\title{
Bin Completion Algorithms for Multicontainer Packing, Knapsack, and Covering Problems
}

\author{
Alex S. Fukunaga \\ FUKUNAGA@AIG.JPL.NASA.GOV \\ Jet Propulsion Laboratory \\ California Institute of Technology \\ 4800 Oak Grove Drive \\ Pasadena, CA 91108 USA \\ Richard E. Korf \\ KORF@CS.UCLA.EDU \\ Computer Science Department \\ University of California, Los Angeles \\ Los Angeles, CA 90095
}

\begin{abstract}
Many combinatorial optimization problems such as the bin packing and multiple knapsack problems involve assigning a set of discrete objects to multiple containers. These problems can be used to model task and resource allocation problems in multi-agent systems and distributed systms, and can also be found as subproblems of scheduling problems. We propose bin completion, a branch-and-bound strategy for one-dimensional, multicontainer packing problems. Bin completion combines a bin-oriented search space with a powerful dominance criterion that enables us to prune much of the space. The performance of the basic bin completion framework can be enhanced by using a number of extensions, including nogood-based pruning techniques that allow further exploitation of the dominance criterion. Bin completion is applied to four problems: multiple knapsack, bin covering, min-cost covering, and bin packing. We show that our bin completion algorithms yield new, state-of-the-art results for the multiple knapsack, bin covering, and min-cost covering problems, outperforming previous algorithms by several orders of magnitude with respect to runtime on some classes of hard, random problem instances. For the bin packing problem, we demonstrate significant improvements compared to most previous results, but show that bin completion is not competitive with current state-of-the-art cutting-stock based approaches.
\end{abstract}

\section{Introduction}

Many NP-hard problems involve assigning some set of discrete objects to multiple containers. In one class of problems, the objective is to pack some items into a set of containers without exceeding the containers' capacities. In a related class of problems, the goal is to cover a set of containers by filling them up to at least some minimal level (quota) using a set of items. When both the containers and the items are modeled as one-dimensional objects (possibly with an associated cost/value function), we refer collectively to these problems as one-dimensional, multicontainer packing and covering problems, or simply multicontainer packing problems.

One example of a multicontainer packing problem is the bin packing problem: Given a set of items (numbers), and a fixed bin capacity, assign each item to a bin so that the sum of the items assigned to each bin does not exceed the bin capacity, and the number of bins 
used is minimized. For example, given the set of items $6,12,15,40,43,82$, and a bin capacity of 100, we can assign 6,12 , and 82 to one bin, and 15, 40, and 43, to another, for a total of two bins. This is an optimal solution to this instance, since the sum of all the items, 198, is greater than 100, and hence at least two bins are required.

Multicontainer packing problems are ubiquitous. They model many important operations research problems such as cargo loading and transport, and also model many artificial intelligence applications, such as the allocation and rationing of resources or tasks among a group of agents. Multicontainer packing problems can often be found embedded as subproblems of more complex, real-world combinatorial optimization problems. For example many constraint programming problems contain "bin packing" or "knapsack" constraints as subproblems (e.g., Shaw, 2004). Such constraints are at the core of many scheduling and resource allocation problems.

In this paper, we propose bin completion, a new algorithm for optimally solving multicontainer packing problems. We begin in Section 1.1 with an overview of four representative, strongly NP-complete, multicontainer problems: (1) the bin packing problem, (2) the multiple knapsack problem, (3) the bin covering problem, and (4) the min-cost covering problem.

In Section 2, we begin by describing the standard, "item-oriented" branch-and-bound framework for these problems. In this traditional approach, items are considered one at a time. Each node in the search corresponds to a decision regarding the assignment of an item to some non-full container. Then, we describe bin completion, an alternative, "binoriented" branch-and-bound strategy with two key features: (1) the nodes in the search tree correspond to complete assignments of items to a single bin, and (2) dominance criteria between assignments of items to bins are used to prune the search. Section 3 describes extensions to the basic bin completion framework that improve search efficiency, as well as the runtime and memory usage at each node.

In Sections 4-7, we explore the application of the bin completion framework to four specific, one-dimensional multicontainer packing problems. For each problem, we review the previous work on algorithms that optimally solve the problem, detail our bin completion algorithm for the problem, and provide an empirical comparison with the previous state-ofthe-art algorithm. We apply bin completion to the multiple knapsack problem in Section 4, and show that our bin completion solver significantly outperforms Mulknap (Pisinger, 1999), the previous state-of-the-art algorithm. The min-cost covering problem (also called the liquid loading problem) was the first problem for which Christofides, Mingozzi, and Toth (1979) proposed an early variant of the bin completion approach. In Section 5, we show that our new bin completion algorithm significantly outperforms the earlier algorithm by Christofides et al. In Section 6, we apply bin completion to the bin covering problem (also known as the dual bin packing problem). We show that our bin completion algorithm significantly outperforms the previous state-of-the-art algorithm by Labbé, Laporte, and Martello (1995). In Section 7, we apply our extended bin completion algorithm to the bin packing problem. Although our initial results were promising (Korf, 2002, 2003), our best bin completion solver is not competitive with the current state of the art, which is a recent branch-and-price approach based on a cutting-stock problem formulation. Section 8 concludes with a discussion and directions for future work. 


\subsection{One-Dimensional, Multicontainer Packing Problems}

We consider the class of multicontainer packing problems: Given a set of items, which must be assigned to one or more containers ("bins"), each item can be assigned to at most one container. Each item $j$ has a weight $w_{j}$ associated with it. Depending on the problem, each item $j$ may also have a profit or cost $p_{j}$ associated with it. We assume that item weights and containers are one-dimensional. For some real-world applications, such as continuous call double auctions (Kalagnanam, Davenport, \& Lee, 2001), this model can be applied directly. For other applications, such as the lot-to-order matching problem, the onedimensional model is an approximation (Carlyle, Knutson, \& Fowler, 2001). We consider two types of containers: (1) Containers with a capacity, where the sum of the weights of the items assigned to a container cannot exceed its capacity, and (2) containers with a quota, where the sum of the weights of the items assigned to a container must be at least as large as the quota. When there is only a single container, we have the well-known 0-1 knapsack problem. See the recent text by Kellerer, Pferschy, and Pisinger (2004) for an overview of work on the 0-1 Knapsack problem and its variants. In this paper, we focus on four one-dimensional, multicontainer packing problems (1) bin packing, (2) multiple knapsack, (3) bin covering, and (4) min-cost covering.

\subsubsection{The Bin Packing Problem}

In the bin packing problem, the goal is to pack $n$ items with weights $w_{1}, \ldots, w_{n}$ into bins of capacity $c$ such that all items are packed into the fewest number of bins, and the sum of the weights of the items in each bin is no greater than the capacity. Classical applications of bin packing include the classic vehicle/container loading problem (Eilon \& Christofides, 1971), as well as memory/storage allocation for data. The minimal number of agents required to carry out a set of tasks in a multiagent planning problem can be modeled as a bin packing problem.

More formally, the bin packing problem can be formulated as the integer program:

$$
\begin{aligned}
& \operatorname{minimize} \sum_{i=1}^{n} y_{i} \\
& \text { subject to: } \sum_{j=1}^{n} w_{j} x_{i j} \leq c y_{i}, \quad i=1, \ldots, n \\
& \sum_{i=1}^{n} x_{i j} \leq 1, \quad j=1, \ldots, n \\
& x_{i j} \in\{0,1\} \quad i=1, \ldots, n, j=1, \ldots, n \\
& y_{i} \in\{0,1\}, \quad i=1, \ldots, n
\end{aligned}
$$

where $y_{i}$ represents whether the $i$ th bin is used or not $\left(y_{i}=1\right.$ if any items are assigned to bin $i, y_{i}=0$ otherwise), and $x_{i j}=1$ if item $j$ is assigned to bin $i$, and 0 otherwise. Constraint 2 ensures that the capacity is not violated for each bin that is instantiated, and constraint 3 ensures that items are assigned to at most one bin. 
In this standard formulation, we assume that all bins have the same capacity. However, this assumption is not restrictive, since instances where bins with different capacities (nonuniform bins) can be modeled by introducing additional items and constraints.

\subsubsection{The 0-1 Multiple Knapsack Problem}

Consider $m$ containers with capacities $c_{1}, \ldots, c_{m}$, and a set of $n$ items, where each item has a weight $w_{1}, \ldots, w_{n}$ and profit $p_{1}, \ldots, p_{n}$. Packing the items in the containers to maximize the total profit of the items, such that the sum of the item weights in each container does not exceed the container's capacity, and each item is assigned to at most one container is the 0-1 Multiple Knapsack Problem, or MKP.

The MKP is a natural generalization of the 0-1 Knapsack Problem where there are $m$ containers of capacities $c_{1}, c_{2}, \ldots c_{m}$. Let the binary decision variable $x_{i j}$ be 1 if item $j$ is placed in container $i$, and 0 otherwise. Then the 0-1 Multiple Knapsack Problem can be formulated as:

$$
\begin{aligned}
& \operatorname{maximize} \sum_{i=1}^{m} \sum_{j=1}^{n} p_{j} x_{i j} \\
& \text { subject to: } \sum_{j=1}^{n} w_{j} x_{i j} \leq c_{i}, \quad i=1, \ldots, m \\
& \sum_{i=1}^{m} x_{i j} \leq 1, \quad j=1, \ldots, n \\
& x_{i j} \in\{0,1\} \quad \forall i, j .
\end{aligned}
$$

Constraint 7 encodes the capacity constraint for each container, and constraint 8 ensures that each item is assigned to at most one container.

The MKP has numerous applications, including task allocation among a group of autonomous agents in order to maximize the total utility of the tasks executed (Fukunaga, 2005), continuous double-call auctions (Kalagnanam et al., 2001), multiprocessor scheduling (Labbé, Laporte, \& Martello, 2003), vehicle/container loading (Eilon \& Christofides, 1971), and the assignment of files to storage devices in order to maximize the number of files stored in the fastest storage devices (Labbé et al., 2003). A special case of the MKP that has been studied in its own right is the Multiple Subset-Sum Problem (MSSP), where the profits of the items are equal to their weights, i.e., $p_{j}=w_{j}$ for all $j$ (e.g., Caprara, Kellerer, \& Pferschy, 2000b 2000a,2003). An application of the MSSP is the marble cutting problem, where given $m$ marble slabs, the problem is to decide how to cut the slabs into sub-slabs (each sub-slab is then further processed into a product) in order to minimize the total amount of wasted marble.

\subsubsection{Bin COVERING}

Suppose we have $n$ items with weights $w_{1}, \ldots, w_{n}$, and an infinite supply of identical containers with quota $q$. The bin covering problem, also known as the dual bin packing problem is to pack the items into containers such that the number of containers that contain sets of items whose sums are at least $q$ is maximized. That is, the goal is to distribute, or ration 
the items among as many containers as possible, given that the containers have a specified quota that must be satisfied. Note that the total weight of the items placed in a container can be greater than $q$ (we assume infinite capacity, although assigning an additional item to a bin whose quota is already satisfies is clearly suboptimal).

More formally, the bin covering problem can be formulated as the integer program:

$$
\begin{array}{rr}
\operatorname{maximize} & \sum_{i=1}^{n} y_{i} \\
\text { subject to: } \sum_{j=1}^{n} w_{j} x_{i j} \geq q y_{i}, & i=1, \ldots, n \\
& \sum_{i=1}^{n} x_{i j} \leq 1, \\
& \\
x_{i j} \in\{0,1\} & j=1, \ldots, n \\
y_{i} \in\{0,1\}, & i=1, \ldots, n, j=1, \ldots, n \\
& i=1, \ldots, n
\end{array}
$$

where $y_{i}$ represents whether the quota on the $i$ th bin is satisfied $\left(y_{i}=1\right)$ or not $\left(y_{i}=0\right)$, and $x_{i j}=1$ if item $j$ is assigned to bin $i$, and 0 otherwise. Constraint 11 ensures that the quota is satisfied for each bin that is instantiated, and constraint 12 ensures that items are assigned to at most one bin.

Bin covering is a natural model for resource or task allocation among multiple agents where the goal is to maximize the number of agents who achieve some quota. It is also models industrial problems such as: (1) packing peach slices into cans so that each can contains at least its advertised net weight in peaches, and (2) breaking up monopolies into smaller companies, each of which is large enough to be viable (Assmann, Johnson, Kleitman, \& Leung, 1984). Another application of bin covering is the lot-to-order matching problem in the semiconductor industry, where the problem is to assign fabrication wafer lots to customer orders of various sizes (Carlyle et al., 2001).

\subsubsection{Min-Cost Covering Problem (Liquid Loading Problem)}

We define the Min-Cost Covering Problem (MCCP) as follows. Given a set of $m$ bins with quotas $\left\{q_{1}, \ldots, q_{m}\right\}$, and a set of $n$ items with weights $w_{1}, \ldots, w_{n}$ and costs $p_{1}, \ldots, p_{n}$, assign some subset of the items to each bin such that (1) each item is assigned to at most one bin, (2) the sum of the weights of the items assigned to each bin is at least the bin's quota (i.e., the bin is covered, as in bin covering), and (3) the total cost of all the items that are assigned to a bin is minimized. This problem has also been called the liquid loading problem (Christofides et al., 1979), because it was originally motivated by the following application: Consider the disposal or transportation of $m$ different liquids (e.g., chemicals) that cannot be mixed. If we are given $n$ tanks of various sizes, each with some associated cost, the problem is to load the $m$ liquids into some subset of the tanks so as to minimize the total cost. Note that here, the "liquids" correspond to containers, and the "tanks" correspond to the items.

Other applications of the MCCP include: (1) the storage of different varieties of grain in different silos, where different types of grains cannot be mixed, (2) storage of food types 
in freezer compartments, (3) a trucking firm which distributes its trucks (of different sizes) among customers with no mixing of different customer's orders on any truck, and (4) storage of information in storage devices (e.g., sensitive customer data that must be segregated between physical filing cabinets or file servers). A closely related problem is the segregated storage problem (Neebe, 1987; Evans \& Tsubakitani, 1993).

More formally, the MCCP can be formulated as the integer program:

$$
\begin{aligned}
& \operatorname{minimize} \sum_{i=1}^{m} \sum_{j=1}^{n} p_{j} x_{i j} \\
& \text { subject to: } \sum_{j=1}^{n} w_{j} x_{i j} \geq q_{i}, \quad i=1, \ldots, m \\
& \sum_{i=1}^{m} x_{i j} \leq 1, \quad j=1, \ldots, n \\
& x_{i j} \in\{0,1\} \quad \forall i, j .
\end{aligned}
$$

The binary variable $x_{i j}$ represents whether item $j$ is assigned to container $i$. Constraint 16 ensures that the quotas of all the bins are satisfied, and constraint 17 ensures that each item is assigned to at most one bin.

\subsubsection{A TAXONOMY OF MULTiCONTAINER PROBLEMS}

Table 1 summarizes the two key, defining dimensions of the four multicontainer problems we study in this paper. One key dimension is whether the problem involves packing a set of items into containers so that the container capacity is not exceeded (packing), or whether the problem requires satisfying the quota associated with a container (covering). Another key dimension is whether all of the items must be assigned to bins, or whether only a subset of items are selected to be assigned to bins. A third dimension (not shown in the table) is the set of item attributes (e.g., weight, profit/cost). Bin packing and bin covering are single-attribute problems (items have weight only), while the multiple knapsack and min-cost covering problems are two-attribute problems (items have weight and profit/cost).

We focus on the bin packing, bin covering, MKP, and MCCP problems because we believe that these are in some sense the most "basic" multicontainer problems. Many combinatorial optimization problems can be viewed as extensions of these problems with additional constraints. For example, the generalized assignment problem can be considered a generalization of a MKP with a more complex profit function.

\begin{tabular}{|l|l|l|}
\hline & assign all items to bins & assign subset of items to bins \\
\hline packing & bin packing & multiple knapsack \\
\hline covering & bin covering & min-cost covering \\
\hline
\end{tabular}

Table 1: Characterizing multicontainer problems. 


\section{Bin Completion}

The multiple-knapsack problem, bin covering problem, min-cost covering problem, and bin packing problem are all strongly NP-complete (proofs by reduction from 3-PARTITION, e.g. Martello \& Toth, 1990; Fukunaga, 2005). Thus, these problems cannot be solved by a polynomial or pseudo-polynomial time algorithm unless $P=N P$, and the state-of-theart approach for optimally solving to these problems is branch-and-bound. In contrast, the single-container 0-1 Knapsack problem and subset sum problem are only weakly $N P$ complete, and can be solved in pseudopolynomial time using dynamic programming algorithms (Kellerer et al., 2004).

In this section, we begin by describing the standard item-oriented branch-and-bound approach to solving multi-container problems. We then describe bin completion, an alternate, bin-oriented strategy. For clarity and simplicity of exposition, we detail the algorithms in the context of the bin packing problem.

\subsection{Item-Oriented Branch-and-Bound}

The standard approach to solving multi-container problems is an item-oriented branch-andbound strategy. Suppose we have a bin packing problem instance where the bin capacity is 100 , and we have 7 items with weights $83,42,41,40,12,11$, and 5 . We perform a branchand-bound procedure to search the space of assignments of items to bins, where each node in the branch-and-bound search tree corresponds to an item, and the branches correspond to decisions about the bin in which to place the item. Assuming that we consider the items in order of non-increasing weight, we first place the 83 in a bin, resulting in the set of bins $\{(83)\}$. Next, we consider the 42 . If we put it in the same bin as the 83 , resulting in $\{(83,42)\}$, then we exceed the bin capacity. The other possibility is to put the 42 in a new bin, resulting in $\{(83),(42)\}$. This is the only feasible choice at this node. Next, the 41 is assigned to a bin. There are three possible places to assign the 41: (a) in the same bin as the 83 , resulting in $\{(83,41),(42)\}$, (b) in the same bin as the 42 , resulting in $\{(83),(42,41)\}$, and (c) in a new bin of its own, resulting in $\{(83),(42),(41)\}$. Option (a) exceeds bin the capacity of the first bin and is infeasible. Options (b) and (c) are feasible choices, so we branch on this item assignment, resulting in two subproblems, to which the search procedure is recursively applied. Figure 1 illustrates a portion of this search space (we show only the feasible nodes).

This branch-and-bound procedure is item-oriented because at each node, we decide upon the placement of a particular item. Upper and lower bounding techniques specific to the problem can be applied to make this basic depth-first strategy more efficient. Itemoriented branch-and-bound appears to be the most "natural" strategy for multicontainer problems. The seminal paper by Eilon and Christofides (1971), which was the first paper to address optimal solutions for multi-container problems, proposed an item-oriented branchand-bound strategy. Most of the work in the literature on algorithms for optimally solving multi-container problems have relied upon this item-oriented strategy. 


\section{(83)}

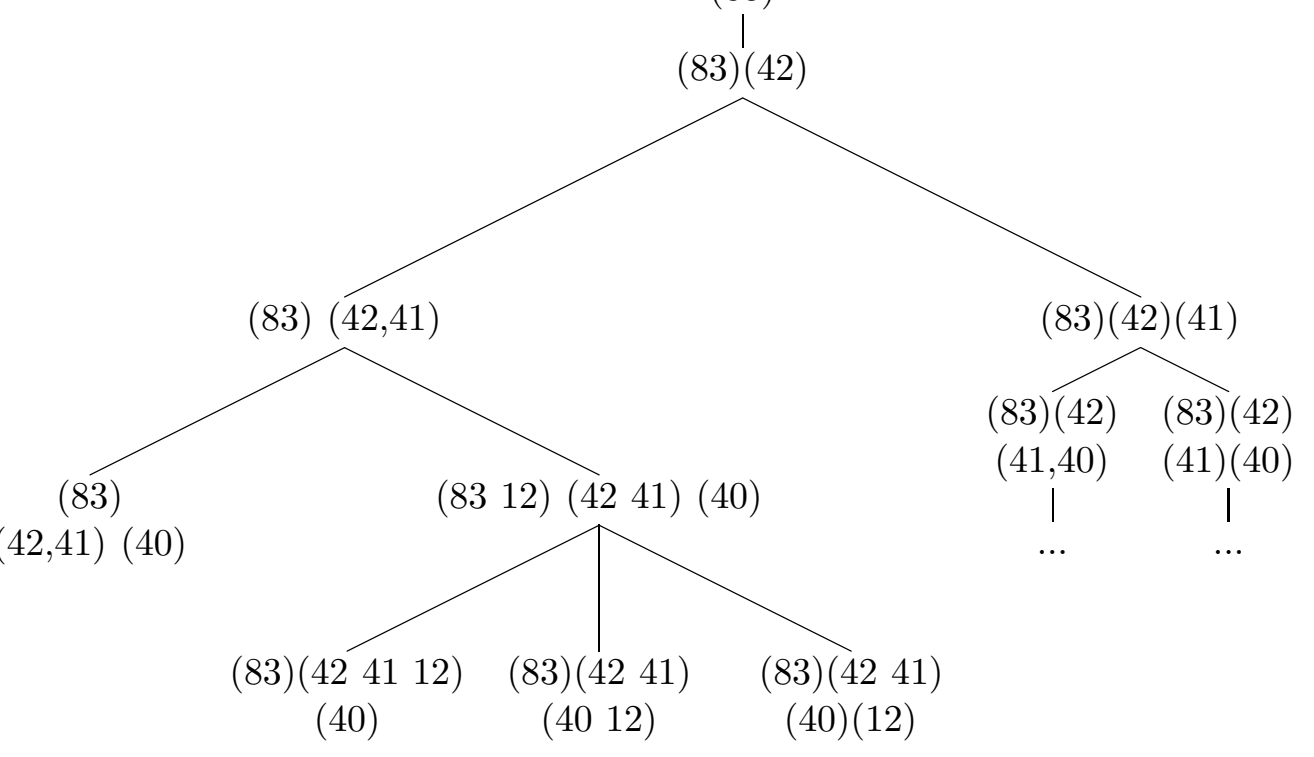

Figure 1: Partial, item-oriented search space for a bin packing instance with capacity 100 and items $\{83,42,41,40,12,11,5\}$. Each node corresponds to a decision about which existing or new bin an item is assigned to. Items are considered in nonincreasing order of weight.

\subsection{Bin Completion, a Bin-Oriented Branch-and-Bound Strategy}

An alternate problem space for solving multi-container problems is bin-oriented, where nodes correspond to decisions about which remaining item(s) to assign to the current bin.

A bin assignment $B=\left(\right.$ item $_{1}, \ldots$, item $\left._{k}\right)$ is a set of all the items that are assigned to a given bin. Thus, a valid solution to a bin packing problem instance consists of a set of bin assignments, where each item appears in exactly one bin assignment. A bin assignment is feasible with respect to a given bin capacity $c$ if the sum of its weights does not exceed c. Otherwise, the bin assignment is infeasible. The definition of feasibility is the same for the MKP; however, for bin covering and the MCCP, we define a bin assignment as feasible with respect to a bin quota $q$ if the sum of its weights is at least $q$. Given a set of $k$ remaining items, we say that a bin assignment $S$ is maximal with respect to capacity $c$ if $S$ is feasible, and adding any of the $k$ remaining items would make it infeasible. Similarly, for bin covering and the MCCP, a feasible bin assignment is minimal with respect to quota $q$ if removing any item would make it infeasible. For brevity, in the rest of this paper, we omit the qualification "with respect to a given capacity/quota" when it is unnecessary (for example, in bin packing and bin covering, all bins have the same capacity/quota so no qualification is necessary).

Bin completion (for bin packing) is a bin-oriented branch-and-bound algorithm in which each node represents a maximal, feasible bin assignment. Rather than assigning items one at a time to bins, bin completion branches on the different maximal, feasible bin assignments. The nodes at a given level in a bin completion search tree represent different maximal, 


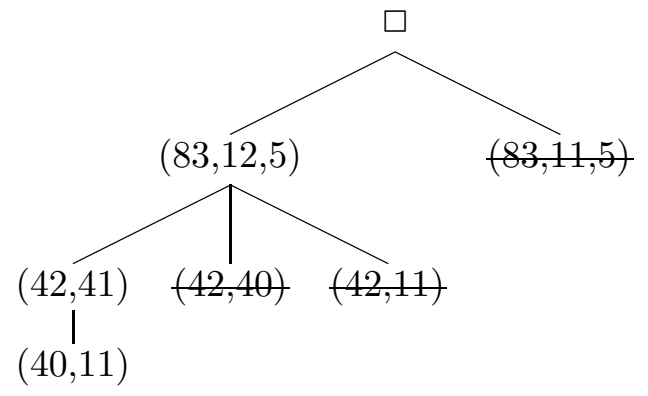

Figure 2: Bin-completion search space for a bin packing instance with capacity 100 and items $\{83,42,41,40,12,11,5\}$. Each node represents a maximal, feasible bin assignment for a given bin. Bin assignments shown with a e.g., (83,11,5), are pruned due to the dominance criterion described in Section 2.3.

feasible bin assignments that include the largest remaining item. The nodes at the next level represent different maximal, feasible bin assignments that include the largest remaining item, etc. The reason that we restrict sibling bin assignments in the bin packing search tree to have the largest remaining number in common is to eliminate symmetries that are introduced by the fact that all bins have the same capacity in our bin packing fomulation. Thus, the depth of any branch of the bin completion search tree corresponds to the number of bins in the partial solution to that depth. Figure 2 shows an example of a bin completion search tree.

\subsection{Dominance Criteria for Bin Assignments}

The key to making bin completion efficient is the use of a dominance criterion on the feasible bin assignments that requires us to only consider a small subset of them.

Bin comletion (for bin packing) only considers maximal, feasible assignments, because it is clear that non-maximal assignments are dominated by maximal assignments, i.e., assigning a non-maximal assignment to a bin cannot lead to a solution with fewer bins than assigning a maximal assignment. For example, if the current bin has 20 units of remaining space and the remaining items are $\{15,30,40,60\}$, it is always better to add the 15 to the current bin, because we must eventually pack the 15 somewhere. We now formalize this notion of dominance and describe more powerful dominance criteria that significantly prune the search space.

Definition 1 (Dominance) Given two feasible bin assignments $F_{1}$ and $F_{2}, F_{1}$ dominates $F_{2}$ if the value of the optimal solution which can be obtained by assigning $F_{1}$ to a bin is no worse than the value of the optimal solution that can be obtained by assigning $F_{2}$ to the same bin.

If we can show that a bin assignment $B$ is dominated by another bin assignment $A$, then we can prune the search tree under $B$. Maximality is a trivial dominance criterion for bin completion. If a feasible bin assignment $B$ is not maximal, then by definition, it must be a 
subset of a maximal, feasible subset $A$, and $B$ is clearly dominated by $A$. We now consider more powerful dominance criteria.

Suppose we have a bin packing instance with bin capacity 100 and items $\{96,3,4,80,15,12\}$. The sets $(96,3)$ and $(96,4)$ are both maximal, feasible bin assignments. If we choose the bin assignment $(96,3)$, the remaining subproblem has the unassigned items $\{80,15,12,4\}$. On the other hand, if we choose the bin assignment $(96,4)$, the remaining subproblem is $\{80,15,12,3\}$. Clearly, the optimal solution to the subproblem $\{80,15,12,4\}$ must use at least as many bins as the optimal solution to the subproblem $\{80,15,12,3\}$. In other words, the optimal solution in the subtree under the node $(96,4)$ is at least as good as the optimal solution in the subtree under $(96,3)$, and therefore there is no need to search under $(96,3)$ because the bin assignment $(96,4)$ dominates the bin assignment $(96,3)$.

Christofides, Mingozzi, and Toth first proposed a more general form for this dominance criterion in the context of the min-cost covering problem (Christofides et al., 1979). We have reformulated their criterion in terms of the bin packing problem:

Proposition 1 (CMT Dominance for Bin Packing) Given two feasible sets $A$ and $B$, $A$ dominates $B$ if: (1) $|A| \geq|B|$ and (2) there exists a one-to-one (but not necessarily onto) mapping $\pi$ from $B$ to $A$ such that each item $b \in B$, is mapped to an element of $A$ with a weight that is greater than or equal to the weight of $b$, i.e., $w(b) \leq w(\pi(b)){ }^{1}$

In other words, if for each element $b$ in a feasible bin assignment $B$, there is a corresponding item $a$ of a feasible bin assignment $A$ such that $b \leq a$, then $A$ dominates $B$. The reason is as follows: consider a bin packing solution $S$, where a single bin is assigned the items in $B$. All of the items must be assigned to some bin, so the items $a \in A$ are assigned to some other bin(s). For each item in $B$, we can swap the corresponding item in $A$, and the resulting solution $S^{\prime}$ will be feasible, and have no more bins than $S$. For example, consider a bin packing instance with the items $\{10,9,8,7,6\}$ and bin capacity 20 . The bin assignment $A=(10,8)$ dominates the bin assignment $B=(9,7)$, because we can map the 9 to the 10 and map the 7 to the 8 .

Martello and Toth (1990) proposed a more powerful dominance criterion that subsumes the CMT criterion. Consider a bin packing instance with items $\{6,4,2,1, \ldots\}$ and capacity 10. The assignment $(6,4)$ dominates the assignment $(6,2,1)$ because given any solution with the assignment $(6,2,1)$, we can swap the 2 and 1 with the 4 , resulting in a solution with the assignment $(6,4)$ and the same number of bins. The CMT criterion does not account for this. More generally, consider two feasible bin assignments $A$ and $B$. If all the elements of $B$ can be packed into bins whose capacities are the elements of $A$, then set $A$ dominates set $B$. For example, let $A=(20,30,40)$ and let $B=(5,10,10,15,15,25)$. Partition $B$ into the subsets $(5,10),(25)$, and $(10,15,15)$. Since $5+10 \leq 20,25 \leq 30$, and $10+15+15 \leq 40$, set $A$ dominates set $B$. More formally:

Proposition 2 (Martello-Toth Bin Packing Dominance Criterion) Let $A$ and $B$ be two feasible bin assignments. A dominates $B$ if $B$ can be partitioned into $i$ subsets $B_{1}, \ldots B_{i}$ such that each subset $B_{k}$ is mapped one-to-one to (but not necessarily onto) an item $a_{k} \in A$ such that the sum of the weights of the items in $B_{k}$ is less than or equal to the weight of $a_{k}$.

1. Recall that a function $f: X \rightarrow Y$ is one-to-one if for any two distinct elements $x, x^{\prime} \in X, f(x) \neq f\left(x^{\prime}\right)$. A function $f: X \rightarrow Y$ is onto $\mathrm{Y}$ if each element of $Y$ is the image under $f$ of some element in $X$. 
Proof: Suppose we have candidate solution $s$ that assigns $B$ to bin $m$, and all of the bin assignments in $s$ are currently feasible. Let $A$ be a feasible bin assignment such that $B$ can be partitioned into $i$ subsets $B_{1}, \ldots, B_{i}$, and each subset $B_{k}$ can be mapped one-to-one to $a_{k} \in A$, such that the sum of the weights of the items in $B_{k}$ is less than or equal to the weight of $a_{k}$. Consider swapping $B$ with $A$. Each subset $B_{k}$ is swapped with its corresponding element $a_{k}$ (where $a_{k}$ is assigned to bin $d_{k}$ in the original solution $s$ ). Since $A$ is feasible, bin $m$ remains feasible after $A$ and $B$ are swapped. Now consider each of the bins $d_{1}, \ldots d_{i}$ where the subsets $B_{1}, \ldots, B_{i}$ end up after the swaps (in other words, $d_{1}, \ldots d_{i}$ are the bins which contain $a_{1}, \ldots, a_{k}$, respectively, in the original solution $s$ ). Each such target bin $d_{k}$ will remain feasible after the swap, since (1) the bin assignment of $d_{k}$ is feasible prior to the swap, and (2) the sum of the weights of the items in $B_{k}$ is less than or equal to the weight of $a_{k}$. Thus, the resulting solution $s^{\prime}$ after the swap is (a) feasible (all bin assignments are feasible) (b) no worse than the initial solution $s$, and (c) assigns $A$ to bin $m$. Therefore, $A$ dominates $B$.

The Martello-Toth dominance criterion is a generalization of the CMT dominance criterion - the CMT dominance criterion is a special case of the Martello-Toth criterion where we only consider partitions of $B$ into single-element subsets. Thus, any node that would be pruned by the CMT criterion is also pruned by the Martello-Toth criterion, but not vice versa.

Similarly, we can define a dominance criterion for bin covering as follows:

For bin covering (and the MCCP), a bin assignment is feasible with respect to a given bin if the sum of the item weights is greater than or equal to the bin quota $q$.

Proposition 3 (Bin Covering Dominance Criterion) Let $A$ and $B$ be two feasible assignments. $A$ dominates $B$ if $B$ can be partitioned into $i$ subsets $B_{1}, \ldots, B_{i}$ such that each item $a_{k} \in A$ is mapped one-to-one to (but not necessarily onto) a subset $B_{k}$, such that the weight of each $a_{k}$ is less than or equal to the sum of the item weights of the corresponding subset $B_{k}$ (i.e., $B_{k}$ "covers" $a_{k}$ ).

Proof: Suppose we have a solution $s$ that assigns $B$ to bin $m$, and all of the bin assignments in $s$ are currently feasible. Let $A$ be a feasible bin assignment such that $B$ can be partitioned into $i$ subsets $B_{1}, \ldots, B_{i}$, and each item $a_{k} \in A$ can be mapped one-to-one to a subset $B_{k}$, such that the weight of $a_{k}$ is less than or equal to the sum of the weights of the items in $B_{k}$. Consider swapping $B$ with $A$. Each subset $B_{k}$ is swapped with its corresponding element $a_{k}$ (where $a_{k}$ is assigned to bin $d_{k}$ in the original solution $s$ ). Since $A$ is feasible, bin $m$ remains feasible after $A$ and $B$ are swapped. Now consider each of the bins $d_{1}, \ldots, d_{|A|}$ where the subsets $B_{k}$ end up after the swaps. In other words, $d_{1}, \ldots, d_{|A|}$ are the bins which contain $a_{1}, \ldots, a_{k}$, respectively, in the original solution $s$. Each such target bin $d_{k}$ will remain feasible after the swap, since (1) the bin assignment of $d_{k}$ is feasible prior to the swap, and (2) the sum of the weights of the items in $B_{k}$ is greater than or equal to the weight of $a_{k}$ (and thus the quota of bin $d_{k}$ will continue to be satisfied). Thus, the resulting solution $s^{\prime}$ after the swap is (a) feasible (all bin assignments are feasible) (b) no worse than the initial solution $s$, and (c) assigns $A$ to bin $m$. Therefore, $A$ dominates $B$.

The dominance criteria for the MKP and MCCP are similar to the dominance criteria for bin packing and bin covering, respectively, except that we must also take into consideration the profits/costs. The proofs are similar to the proofs for bin packing and bin covering. 
Proposition 4 (MKP Dominance Criterion) Let $A$ and $B$ be two assignments that are feasible with respect to capacity $c$. $A$ dominates $B$ if $B$ can be partitioned into $i$ subsets $B_{1}, \ldots, B_{i}$ such that each subset $B_{k}$ is mapped one-to-one to (but not necessarily onto) $a_{k}$, an element of $A$, and for all $k \leq i,(1)$ the weight of $a_{k}$ is greater than or equal the sum of the item weights of the items in $B_{k}$, and (2) the profit of item $a_{k}$ is greater than or equal to the sum of the profits of the items in $B_{k}$.

Proposition 5 (MCCP Dominance Criterion) Let $A$ and $B$ be two assignments that are feasible with respect to quota $q$. A dominates $B$ if $B$ can be partitioned into $i$ subsets $B_{1}, \ldots, B_{i}$ such that each item $a_{k} \in A$ is mapped one-to-one (but not necessarily onto) to a subset $B_{k}$, and for each $a_{k} \in A$ and its corresponding subset $B_{k}$, (1) the weight of $a_{k}$ is less than or equal to the sum of the item weights of the items in $B_{k}$, and (2) the cost of item $a_{k}$ is less than or equal to the sum of the cost of the items in $B_{k}$.

The CMT dominance criterion for the MCCP originally proposed in (Christofides et al., 1979) is a special case of Proposition 5, where $\left|B_{k}\right|=1$ for all $k$.

Note that in the "packing" problems such as the MKP and bin packing, the dominance criteria require that the subsets of the dominated assignment are packed into the items of the dominating assignment. In contrast, with the "covering" problems such as the MCCP and bin covering, the dominance criteria requires that the subsets of the dominated assignment $(B)$ cover the items in the dominating assignment $(A)$.

\subsubsection{Bin-Oriented Branch-And-Bound + Dominance $=$ Bin Completion}

At this point, we have defined the salient features of the bin completion approach to solving multicontainer packing and knapsack problems:

- A bin-oriented branch-and-bound search where the nodes correspond to maximal (or minimal), feasible bin assignments; and

- The exploitation of a dominance criterion among bin assignments to prune the search space.

The first instance of a bin completion algorithm that we are aware of is the Christofides, Mingozzi, and Toth algorithm for the min-cost covering problem in 1979 (Christofides et al., 1979), which used the CMT criterion described above. However, as far as we know, no further research was done with bin completion algorithms until our work on bin completion for bin packing (Korf, 2002).

The Martello-Toth dominance criterion was proposed by Martello and Toth (1990), as a component of the Martello-Toth Procedure (MTP), a branch-and-bound algorithm for bin packing. However, the MTP branch-and-bound algorithm is item-oriented, and they only exploit this dominance property in a limited way. In particular, they take each remaining element $x$, starting with the largest element, and check if there is a single assignment of $x$ with one or two more elements that dominates all feasible sets containing $x$. If so, they place $x$ with those elements in the same bin, and apply the reduction to the remaining subproblem. They also use dominance relations to prune some element placements as well.

Another earlier instance of a bin completion algorithm is the BISON algorithm for bin packing by Scholl, Klein, and Jürgens (1997). BISON uses the following, very limited form 
of the Martello-Toth dominance criterion: if a bin assignment has one or more items that can be replaced by a single free item without decreasing the sum, then this assignment is dominated. It is interesting that despite the fact that the basic idea of bin-oriented search with dominance-based pruning was demonstrated by Christofides, Mingozzi, and Toth, both the MTP and BISON only use a limited form of the Martello-Toth dominance criterion, and the two ideas were not successfully integrated until our work. ${ }^{2}$ Presumably, the reason is that it is not trivial to generate undominated bin assignments efficiently.

\subsection{Generating Undominated Bin Assignments}

A key component in bin completion is the efficient generation of undominated bin assignments. An obvious approach is to generate all feasible bin assignments, then apply the dominance tests to eliminate the dominated assignments. However, this is impractical, because the number of feasible assignments is exponential in the number of remaining items, and the memory and time required to generate and store all assignments would be prohibitive. We now describe an algorithm that generates all and only undominated bin assignments, which enables the efficient implementation of bin completion.

We can generate all subsets of $n$ elements by recursively traversing a binary tree. Each internal node corresponds to an item, where the left branch corresponds to the subsets that include the item, and the right branch corresponds to the subsets that do not include the item. Thus, each leaf node represents an individual subset. Note that this binary tree is the search space for this subproblem (generating undominated bin assignments), and is distinct from the search space of the higher level bin-completion algorithm (i.e.. the space of undominated maximal assignments).

Given $n$ elements and a container capacity $c$, the feasible bin assignments are the subsets of the $n$ elements, the sum of whose weights do not exceed $c$. The recursive traversal described above for generating all subsets can be modified to generate only feasible assignments as follows: At each node in the tree, we keep track of the sum of items that we have committed to including in the subset (i.e., the sum of the weights of the items for which we have taken the left branch). During the recursive traversal of the tree, we pass a parameter representing the remaining capacity of the bin. Each time a left branch is taken (thereby including the item), we reduce the remaining capacity by the item weight. If the remaining capacity drops to zero or less, we prune the tree below the current node, since we have equaled or exceeded the container capacity. Thus, this algorithm generates only feasible bin assignments.

Now, we further extend the algorithm to generate only the undominated, feasible bin assignments. Suppose that we have a feasible set $A$ whose sum of weights is $t$. The excluded items are all items that are not in $A$. Set $A$ will be dominated (with respect to the MartelloToth dominance criterion) if and only if it contains any subset whose sum $s$ is less than or equal to an excluded item $x$, such that replacing the subset with $x$ will not exceed the bin capacity $c$. This will be the case if and only if there exists an excluded item $x$ and a subset with weight sum $s$ such that $t-s+x \leq c$. Therefore, to check if $A$ is undominated, we enumerate each possible subset of its items, and for each subset, we compare it against each

2. As shown in (Korf, 2002, 2003), bin-oriented search using the full Martello-Toth dominance criterion results in dramatic speedups compared to the MTP. 
excluded number $x$ to verify that $t-s+x>c$, where $s$ is the sum of the item weights of the subset. If so, then $A$ is undominated, and we store $A$ in a list of undominated assignments; otherwise, $A$ is dominated. Further optimizations can be found in (Korf, 2003).

This algorithm generates feasible bin assignments and immediately tests them for dominance, so it never stores multiple dominated bin assignments. Furthermore, the dominance test is done by comparing the included elements to excluded elements, and does not involve any comparison between a candidate bin assignment and previously generated bin assignments. Therefore, the memory required for dominance testing is linear in the number of items. In contrast, a method that depends on comparisons between candidate sets, such as the earlier algorithm described in (Korf, 2002), requires memory that is linear in the number of undominated bin assignments, which is potentially exponential in the number of items. The ability to incrementally generate undominated bin assignments using linear space without having to store all of the undominated assignments enables the hybrid incremental branching strategy, described in Section 3.4.

\section{Extensions to Bin Completion}

We now describe some extensions to bin completion that significantly improve search efficiency. Again, for clarity, we describe the algorithms mostly in the context of bin packing, but as with the basic bin completion algorithm, these extensions can be adapted straightforwardly to the multiple knapsack, bin covering, and min-cost covering problems.

\subsection{Nogood Pruning (NP)}

Nogood pruning prunes redundant nodes in the bin completion search tree by detecting symmetries. Since we need to refer to specific bins, we extend our notation for bin assignment. Let $(A)_{d}$ denote a bin at depth $d$ that is assigned the elements in $A$. Thus, $(10,8,2)_{1}$ and $(10,7,3)_{1}$ denote two possible bin assignments for the bin at depth 1 .

Suppose we have an instance with the numbers $\{10,9,8,7,7,3,3,2,2\}$, and bin capacity $\mathrm{c}=20$. After exhausting the subproblem below the assignment $(10,8,2)_{1}$, and while exploring the subproblem below the assignment $(10,7,3)_{1}$, assume we find a solution that assigns $(9,8,2)_{2}$. We can swap the pair of items $(8,2)$ from the assignment $(9,8,2)_{2}$ with the pair of items $(7,3)$ from the assignment $(10,7,3)_{1}$, resulting in a solution with $(10,8,2)_{1}$ and $(9,7,3)_{2}$ and the same number of bins. However, we have already exhausted the subtree below $(10,8,2)_{1}$ and we would have found a solution with the same number of bins as the best solution in the subtree below $(9,7,3)_{2}$. Therefore, we can prune the branch below $(9,8,2)_{2}$, because it is redundant (in other words, we have detected that the current partial solution is symmetric to a partial state that has already been exhaustively searched).

More formally, let $\left\{N_{1}, N_{2}, \ldots, N_{m}\right\}$ be a set of sibling nodes in the search tree, and let $\left\{S_{1}, S_{2}, \ldots, S_{m}\right\}$ be the bin assignments for each sibling node, excluding the first item assigned to the bin, which is common to all the sibling nodes. When searching the subtree below node $N_{i}$ for $i>1$, we exclude any bin assignment $B$ that (1) includes all the items in $S_{j}$, and (2) swapping the items in $S_{j}$ from $B$ with the items $S_{i}$ in $N_{i}$ results in two feasible 
bin assignments, for $i>j$. The items in $S_{j}$ become a nogood with respect to nodes deeper in the tree. ${ }^{3}$

If there exists such a bin assignment $B$, then we could swap the items $S_{j}$ from $B$ with the items $S_{i}$ in $N_{i}$, resulting in a partial solution with the bin assignment $S_{i}$ in bin $N_{i}$. However, we have already exhausted the subtree below $N_{i}$, so this is a redundant node and can be pruned.

As the search progresses down the tree, a list of nogoods is maintained, which is the set of bin assignments against which each candidate undominated bin assignment is compared. Given a candidate bin assignment $B$, where the items are sorted according to weight, the current implementation of the test for nogood pruning compares the items against each nogood. Since the items in the nogood sets are also sorted by weight, each comparison takes time linear in the cardinality of $B$. In the worst case, the number of nogoods that we must compare with a candidate assignment at level $d$ corresponds to the number of undominated bin assignments at levels $1, \ldots, d-1$ that are currently on the stack (that is, all of the ancestors and the siblings of the ancestors of the current node). Note that the list of nogoods need not grow monotonically as we go down the search tree. If at any point, a $\operatorname{nogood} N$ is no longer a subset of the set of remaining items, then $N$ has been 'split', and can be removed from the nogood list that is passed down the search tree.

\subsection{Nogood Dominance Pruning (NDP)}

The following nogood dominance pruning (NDP) technique allows even more pruning: Suppose that after exhausting the subproblem below the assignment $(10,8,2)_{1}$, and while exploring the subproblem below the assignment $(10,7,3)_{1}$, we consider the assignment $(9,7,2)_{2}$. We can swap the pair of items $(7,2)$ from bin 2 with the pair of items $(7,3)$ from bin 1 and end up with a solution with $(10,7,2)_{1}$ and $(9,7,3)_{2}$. However, according to the Martello-Toth dominance criterion, $(10,7,2)$ is dominated by $(10,8,2)$, and we have already exhausted the search below the node $(10,8,2)_{1}$, so we can prune the search under $(9,7,2)_{2}$ because it is not possible to improve upon the best solution under $(10,8,2)_{1}$.

In general, given a node with more than one child, when searching the subtree of any child but the first, we don't need to consider assignments that are dominated by a bin assignment in a previously explored child node. More precisely, let $\left\{N_{1}, N_{2}, \ldots, N_{m}\right\}$ be a set of sibling nodes in the search tree, and let $\left\{S_{1}, S_{2}, \ldots, S_{m}\right\}$ be the corresponding sets of items used in the bin assignment at each node. When searching the subtree below node $N_{i}$ for $i>1$, we exclude any bin assignment $A$ for which there exists an assignment $S_{j}$, $j<i$, such that (1) $A$ is dominated by the items in $S_{j}$ (note that an assignment dominates itself), and (2) $A$ can be swapped with $S_{i}$, such that the resulting bin assignments are both feasible. If there exists such a bin assignment $A$, then we could swap the items from $A$ with the items $S_{i}$ in $N_{i}$, resulting in a partial solution with the bin assignment $A$ in bin $N_{i}$, and the same number of bins. However, since $A$ is dominated by $S_{j}$, it means that we are searching a node that is symmetric to one that is dominated by $S_{j}$, and therefore, it is not possible to find a solution better than the best solution under $S_{j}$, so $A$ can be pruned.

3. While the term nogood in the constraint programming literature often refers to an assignment of value(s) to variable(s) that cannot lead to a solution, we use the term to mean an assignment that cannot lead to any solution that is better than a previously discovered solution, similar to the usage in (Focacci \& Shaw, 2002). 
We can also describe nogood dominance pruning in terms of a more general constraint programming formulation, where variables correspond to items and values denote the bins to which they are assigned. Given a partial, $j$-variable solution $x$, nogood dominance pruning tries to show, via swapping of items and dominance checks, that $x$ is in the same equivalence class as another partial solution $x^{\prime}$ such that $\bar{x}_{i}^{\prime}$, the subset of $x^{\prime}$ including the first $i$ variables, is dominated by another partial solution $q$. Thus, if we have exhausted the subtree below $q$ in the search tree, we do not need to search the subtree below $x^{\prime}$.

Nogood dominance pruning is strictly more powerful than nogood pruning. Any node pruned by nogood pruning will be pruned by nogood dominance pruning, but not vice versa. Of course, since NDP must detect dominance relationships as opposed to equivalence relationships, NDP will incur more overhead per node compared to NP. Our current implementation propagates a list of nogood sets along the tree. While generating the undominated completions for a given bin, we check each one to see if it is dominated by any current nogood. If so, we ignore that bin assignment. Our current implementation uses a brute-force algorithm to check for dominance between a candidate bin assignment and each nogood, which in the worst case takes time exponential in the cardinality of the bin assignment (for each nogood).

Since nogood pruning is much less expensive than nogood dominance pruning, we use a combined pruning strategy. Whenever we apply NDP, we actually apply both NP and NDP at each node. First, the candidate bin assignment is checked against a nogood, and nogood pruning is applied. We apply nogood dominance pruning only if the bin assignment was not pruned by nogood pruning. Thus, we never pay the full cost of applying NDP to nodes that can be pruned quickly by NP. As with nogood pruning, NDP requires storing a set of nogoods, and the number of possible nogoods at a particular search depth $d$ in the worst case is the number of undominated bin assignments considered at depths $1, \ldots, d-1$. Note that when using NDP, we do not apply the optimization described above in Section 3.1 for removing nogoods from the nogood list that are passed down the tree when using nogood pruning. The reason is that even if a nogood has been "split" and can no longer prune a bin assignment due to nogood pruning, that nogood may still be able to prune a bin assignment due to nogood dominance pruning.

The size of the nogood list increases with depth, and we compare each bin assignment against each nogood. Therefore, the per-node overhead of NDP increases with depth. This means that pruning at the bottom of the tree (where pruning has the lowest utility) is more expensive than pruning at the top of the tree (where pruning has the highest utility). A simple strategy which address this issue is depth-limited NDP, where NDP is applied only at nodes up to the NDP depth limit $L$. At nodes below the depth limit, only the weaker nogood pruning is applied. In the experiments described in this paper, we did not use this depth-limited strategy because NDP consistently outperformed nogood pruning without having to use depth limits.

\subsection{Related Work in Constraint Programming}

Nogood pruning identifies and prunes nodes by detecting whether the bin assignment for the current node contains a nogood. This is related to other symmetry-breaking techniques proposed in the constraint programming literature. A symmetry partitions the set of pos- 
sible assignments of values to variables into equivalence classes (Gent \& Smith, 2000). The goal of symmetry breaking is to prune nodes that can be mapped to a previously explored node via a symmetry function. Symmetry-breaking approaches introduce constraints during the search that prune symmetric variable assignments (e.g., Gent \& Smith, 2000; Fahle, Schamberger, \& Sellmann, 2001; Focacci \& Milano, 2001). Similarly, our nogood and nogood dominance pruning techniques dynamically introduce constraints that prune variable assignments that cannot lead to a solution that is better than the best solution found so far. Nogood dominance pruning uses the same dynamic nogood recording mechanism as nogood pruning, but goes a step further in detecting dominance relationships based on the nogoods. The general notion of dominance exploited by NDP is more powerful than symmetry, since dominance can be asymmetric, i.e., all symmetries are dominance relationships, but not vice versa.

Our NDP technique is similar to the pruning technique proposed by Focacci and Shaw (2002) for constraint programming, who applied their technique to the symmetric and asymmetric traveling salesperson problem with time windows. Both methods attempt to prune the search by proving that the current node at depth $j$, which represents a partial $j$ variable (container) solution $x$, is dominated by some previously explored $i$-variable partial solution (nogood), $q$. The main difference between the two methods is the approach used to test for dominance. Focacci and Shaw's method extends $q$ to a $j$-variable partial solution $q^{\prime}$ which dominates $x$. They apply a local search procedure to find the extension $q^{\prime}$.

In contrast, our NDP method starts with a partial, $j$-variable solution $x$ and tries to transform it to a partial solution $x^{\prime}$ such that $\bar{x}_{i}^{\prime}$, the subset of $x^{\prime}$ including the first $i$ variables, is dominated by $q$. We do this by swapping the values of the $i$ th and $j$ th variables in $x$ to derive $x^{\prime}$, and testing whether $\bar{x}_{i}^{\prime}$ is dominated by $q$.

For efficiency, the current implementations of both nogood dominance pruning methods are weak, in the sense that if $x$ is dominated by $q$, the procedures will not necessarily detect the dominance. Focacci and Shaw rely on an incomplete, local search to find the extension $q^{\prime}$. Due to the cost of considering the transformations, we only consider transformations involving two variables (containers), but to fully exploit the dominance criterion, we would need to consider transformations involving all variables $i, i+1, \ldots, j$.

\subsection{Reducing the Computation Required Per Node}

An issue with enumerating all undominated completions and applying value ordering is that computing the undominated sets is itself NP-complete. For a multicontainer problem instance where the items have weight $w_{i}$ and average container capacity is $c$, the average number of items that fit in a container is $x=\sum_{i=0}^{n} w_{i} / c$. The time to generate all undominated bin assignments increases with $x$. This is not an issue for bin packing, where problems with large $x$ tend to be easily solved using heuristics such as best-fit decreasing. The solution found by the heuristic often equals the lower bound. Therefore, such instances require no search, and hence there is no need to compute undominated bin assignments. On the other hand, for multiple knapsack and bin covering, we have observed experimentally that it is much less likely that heuristics will match the optimistic bound and allow termination without search, and we have found that for instances with high $x$, the algorithm would not terminate within a reasonable time limit because it was spending an inordinate amount of 


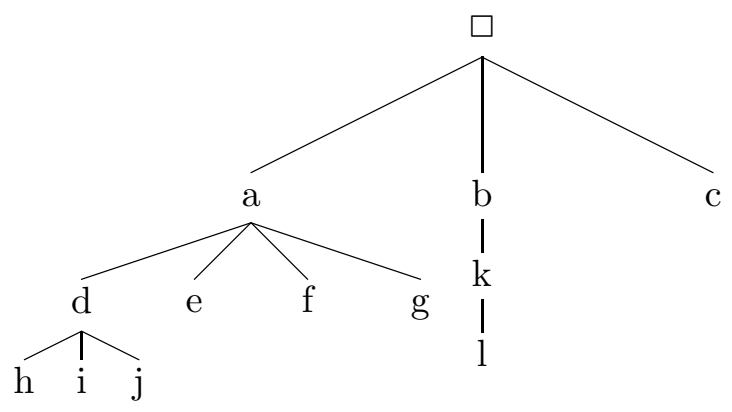

Figure 3: Hybrid Incremental Branching Strategy

time computing the set of undominated completions at each node. In addition, generating all undominated bin assignments may cause us to run out of memory.

An alternative approach is to start to go down the search tree and explore some of the children of a node without first enumerating and sorting all the children. In cases where (a) a good optimistic bound is available, and (b) it takes relatively little search to find an optimal solution, this approach leads to dramatic speedups compared to the original scheme of generating all undominated completions before going further down the search tree. The price we pay for this strategy is that we lose the benefits of ordering all the candidate bin assignments according to the value-ordering heuristic.

To solve this problem, we propose a hybrid incremental branching strategy that generates $h$ children at each node, applies the value-ordering heuristic to these, then recursively calls bin completion on the remaining subproblems. For each node, we first generate $h$ children, and sort them according to the value-ordering heuristic. Then, we explore the subtrees under each of these children. After the subtrees under the first $h$ children are fully explored, then the next $h$ children are generated and their subtrees are explored, and so on.

For example, consider the tree in Figure 3, where the nodes correspond to undominated bin assignments. Assume that this is the complete search tree, and assume for simplicity that there is no value ordering heuristic. The standard bin completion algorithm first generates the children of the root: $a, b$, and $c$. Then, bin completion selects one of the children (say $a$ ), and expands $a$ 's children $(d, e, f, g)$. It then generates the children of $d$, and so on. Thus, the order of node generation is: $a, b, c, d, e, f, g, h, i, j, k, l$. This is a pre-order traversal of the tree. Now consider hybrid incremental branching with width 1 . This corresponds to a standard postorder traversal of the tree, where the order in which nodes are generated is $a, d, h, i, j, e, f, g, b, k, l, c$. Hybrid incremental branching with width 2 generates the nodes in the order $a, b, d, e, h, i, j, f, g, k, l, c$.

\section{The Multiple Knapsack Problem (MKP)}

Given a set of containers and a set of items (characterized by a weight and profit), the objective of the multiple knapsack problem (defined formally in section 1.1.2) is to assign items to containers such that the container capacities are not exceeded, and the sum of the profits of the items assigned to the containers is maximized.

We compared bin completion to two standard algorithms: the state-of-the-art Mulknap algorithm (Pisinger, 1999), as well as the MTM algorithm (Martello \& Toth, 1981). 


\subsection{The MTM Algorithm}

The MTM algorithm of Martello and Toth (1981) is an item-oriented branch-and-bound algorithm. The items are ordered according to non-increasing efficiency (ratio of profit to weight), so that the next item selected by the variable-ordering heuristic for the itemoriented branch-and-bound is the item with highest efficiency that was assigned to at least one container by a greedy bound-and-bound procedure (see below). The branches assign the selected item to each of the containers, in order of non-decreasing remaining capacity.

At each node, an upper bound is computed using a relaxation of the MKP, which is obtained by combining all of the remaining $m$ containers in the MKP into a single container with aggregate capacity $C=\sum_{i=1}^{m} c_{i}$, resulting in the single-container, 0-1 knapsack problem:

$$
\begin{aligned}
& \operatorname{maximize} \sum_{j=1}^{n} p_{j} x_{j}^{\prime} \\
& \text { subject to } \sum_{j=1}^{n} w_{j} x_{j}^{\prime} \leq C, \\
& \qquad x_{j}^{\prime} \in\{0,1\}, j=1, \ldots, n .
\end{aligned}
$$

where the variable $x_{j}^{\prime}$ represents whether item $j$ has been assigned to the aggregated bin. This surrogate relaxed MKP (SMKP) can be solved by applying any algorithm for optimally solving the 0-1 Knapsack problem, and the optimal value of the SMKP is an upper bound for the original MKP. Thus, this upper bound computation is itself solving an embedded, weakly NP-complete (single-container) 0-1 Knapsack problem instance as a subproblem. The SMKP is currently the most effective upper bound for the MKP (details on how the formulation above is derived from an initial surrogate relaxation are in Martello \& Toth, 1981; Kellerer et al., 2004).

At each node, the MTM algorithm applies an extension to branch-and-bound called bound-and-bound. Consider a branch-and-bound procedure for a maximization problem. If the upper (optimistic) bound computed at a node is less than or equal to the best known lower bound for the problem, then the node can be pruned. In the standard branch-andbound approach, the lower bound is simply the objective function value of the best solution found so far.

In bound-and-bound, we attempt to validate the upper bound by applying a fast, heuristic algorithm in order to find a solution whose score equals the upper bound. If such a solution can be found, then it means that we have found the optimal solution under the current node (i.e., the upper bound has been validated), and we can backtrack. On the other hand, if no such solution is found, then we must continue to search under the node. The MTM algorithm applies a greedy heuristic algorithm for the MKP, which involves solving a series of $m$ 0-1 Knapsack problems. First, container $i=1$ is filled optimally using any of the remaining items, and the items used to fill container $i=1$ are removed. Then, container $i=2$ is filled using the remaining items. This process is iterated $m$ times, at which point all containers are filled. 


\subsection{The Mulknap Algorithm}

The previous state-of-the-art algorithm for the MKP is Mulknap (Pisinger, 1999). Like MTM, Mulknap is an item-oriented branch-and-bound algorithm using the SMKP upper bound and bound-and-bound. Mulknap differs from MTM in that it (1) uses a different validation strategy for the bound-and-bound based on splitting the SMKP solution, (2) applies item reduction at each node, and (3) applies capacity tightening at each node.

Like MTM, Mulknap uses a bound-and-bound strategy, but it uses a different approach to validating the upper bound: As described above, the upper bound for a MKP instance can be computed by solving the surrogate relaxation of the MKP, or SMKP, which is a 0-1 Knapsack instance. Suppose we have just computed the optimal solution to the SMKP. Now, suppose we are able to partition the items used in the SMKP into the $m$ containers in the original MKP, such that each item used in the SMKP solution is assigned to some container, and the capacity constraints are not violated. In this case, we have a solution to the original MKP that achieves the upper bound. Details on the capacity tightening and reduction procedures are given by Pisinger (1999).

\subsection{Bin Completion Algorithm for the MKP}

We now describe our bin completion algorithm for the MKP. We apply a depth-first, bin completion branch-and-bound algorithm. Each node in the search tree represents a maximal, feasible bin assignment for a particular bin. Note that in the MKP, bin capacities can vary, so at each node, we consider all undominated bin assignments for the bin. This is in contrast to bin packing 2.2, where (assuming all bins have identical capacity), we can restrict all nodes at the same level to have the same (largest) item.

At each node, an upper bound for the remaining subproblem is computed using the surrogate relaxed MKP (SMKP) bound. The SMKP bound is computed using a straightforward branch-and-bound algorithm with the Martello-Toth U2 upper bound (Martello \& Toth, 1977). Pisinger's R2 reduction procedure (Pisinger, 1999) is applied at each node in order to possibly reduce the remaining problem. Then, we select the bin with smallest remaining capacity (i.e., we use a smallest-bin-first variable ordering heuristic, with ties broken randomly), and its undominated bin assignments are computed and explored using our dominance criterion (Proposition 4). Nogood pruning and nogood dominance pruning are applied as described in Sections 3.1 and 3.2.

The order in which we branch on the undominated children of the current node has a significant impact on the algorithm's performance. At each node, all of the undominated children of the node are generated and ordered using a value ordering heuristic. We evaluated 11 different value ordering heuristics and found that the best performing heuristic overall was the min-cardinality-max-profit ordering, where candidate bin assignments are sorted in order of non-decreasing cardinality and ties are broken according to non-increasing order of profit. Fukunaga (2005) provides further details on experiments evaluating various value and variable ordering strategies. Figure 4 shows an outline of our bin completion algorithm for the MKP. 


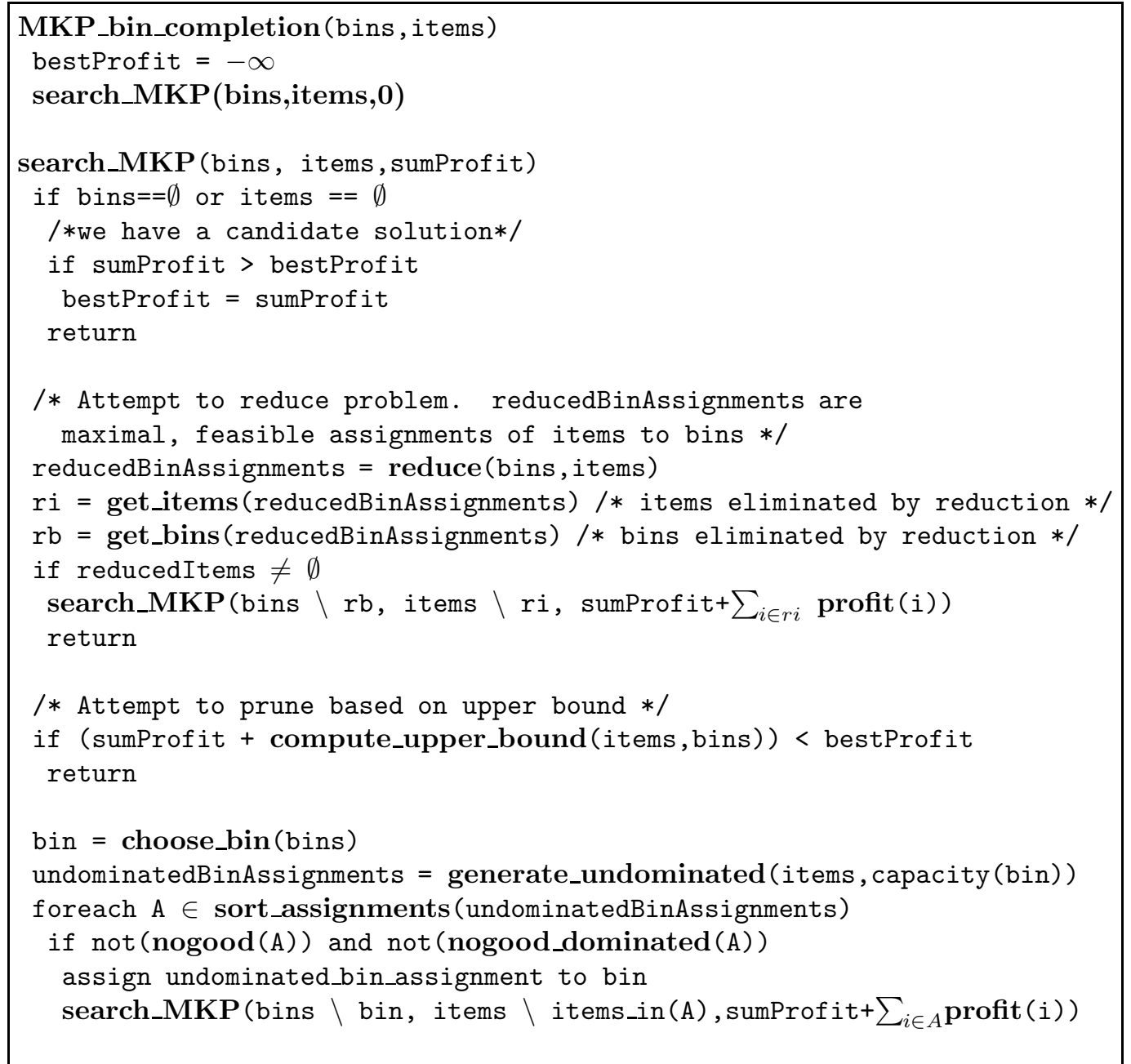

Figure 4: Outline of bin completion for the multiple knapsack problem. compute_lower_bound returns the SMKP upper bound on profit for the remaining subproblem, and reduce applies Pisinger's R2 reduction to eliminate items and bins if possible. choose_bin selects the bin with least remaining capacity, and and sort_assignments sorts the undominated bin assignments in order of non-decreasing cardinality, and ties are broken in order of non-increasing profit. generate_undominated generates undominated bin assignments using the algorithm in (Section 2.4). nogood and nogood_dominated apply nogood pruning (Section 3.1) and nogood dominance pruning (Section 3.2). 


\subsection{Experimental Results}

We evaluated our MKP algorithm using the same four classes of instances used by Pisinger (1999). We considered:

- uncorrelated instances, where the profits $p_{j}$ and weights $w_{j}$ are uniformly distributed in $[\min , \max ]$.

- weakly correlated instances, where the $w_{j}$ are uniformly distributed in [min,max] and the $p_{j}$ are randomly distributed in $\left[w_{j}-(\max -\min ) / 10, w_{j}+(\max -\min ) / 10\right]$ such that $p_{j} \geq 1$,

- strongly correlated instances, where the $w_{j}$ are uniformly distributed in [min,max] and $p_{j}=w_{j}+(\max -\min ) / 10$, and

- multiple subset-sum instances, where the $w_{j}$ are uniformly distributed in [min,max] and $p_{j}=w_{j}$.

The bin capacities were set as follows: The first $m-1$ capacities $c_{i}$ were uniformly distributed in $\left[0.4 \sum_{j=1}^{n} w_{j} / m, 0.6 \sum_{j=1}^{n} w_{j} / m\right]$ for $i=1, \ldots, m-1$. The last capacity $c_{m}$ is chosen as $c_{m}=0.5 \sum_{j=1}^{n} w_{j}-\sum_{i=1}^{m-1} c_{i}$ to ensure that the sum of the capacities is half of the total weight sum. Degenerate instances were discarded as in Pisinger's experiments (1999). That is, we only used instances where: (a) each of the items fits into at least one of the containers, (b) the smallest container is large enough to hold at least the smallest item, and (c) the sum of the item weights is at least as great as the size of the largest container. In our experiments, we used items with weights in the range $[10,1000]$.

\subsubsection{Comparison of Bin Completion With Previous Algorithms}

We now compare bin completion (BC) with Mulknap and MTM. In all experiments described below involving Mulknap, we used Pisinger's Mulknap code, available at his web site $^{4}$, compiled using the gcc compiler with the -03 optimization setting. Likewise, in all experiments described below involving MTM, we used Martello and Toth's Fortran implementation of MTM from (Martello \& Toth, 1990), which was converted to C using f2c so that we could add some instrumentation. Our bin completion code was implemented in Common Lisp. ${ }^{5}$ We have shown experimentally that the choice of programming language added an overhead of approximately a factor of two to our runtimes (see Fukunaga, 2005, Appendix A) compared to GNU C 2.95 with -03 optimization.

For each of the four problem classes, we generated test sets of 30 instances each, where $n=20$, and $m$ was varied between 2 and 10. On each instance, we ran Mulknap, bin completion, and bin completion with nogood dominance pruning. The purpose of this experiment was to observe the behavior of the algorithms as the ratio $n / m$ was varied. The results are shown in Table 2. Each algorithm was given a time limit of 300 seconds to solve each instance. The fail column indicates the number of instances (out of 30) which could not be solved by the algorithm within the time limit. The time and nodes column show the

4. http://www.diku.dk/ pisinger/

5. All of the bin completion solvers described in this paper for the MKP, MCCP, bin covering, and bin packing problems are implemented in Common Lisp. 


\begin{tabular}{|c|c|c|c|c|c|c|c|c|c|c|}
\hline & & \multicolumn{3}{|c|}{ MTM } & \multicolumn{3}{|c|}{ Mulknap } & \multicolumn{3}{|c|}{ Bin Completion+NDP } \\
\hline $\begin{array}{r}\mathrm{m} \\
\text { \# bins }\end{array}$ & $\begin{array}{r}n \\
\text { \# items }\end{array}$ & fail & time & nodes & fail & time & nodes & fail & time & nodes \\
\hline \multicolumn{11}{|c|}{ Uncorrelated Instances } \\
\hline 2 & 20 & 0 & 0.0003 & 201 & 0 & 0.0000 & 1 & 0 & 0.0050 & 55 \\
\hline 3 & 20 & 0 & 0.0017 & 697 & 0 & 0.0000 & 23 & 0 & 0.0063 & 123 \\
\hline 4 & 20 & 0 & 0.0063 & 1530 & 0 & 0.0030 & 306 & 0 & 0.0023 & 112 \\
\hline 5 & 20 & 0 & 0.0767 & 34800 & 0 & 0.0387 & 2977 & 0 & 0.0033 & 212 \\
\hline 6 & 20 & 0 & 0.2343 & 100387 & 0 & 0.4063 & 36057 & 0 & 0.0050 & 403 \\
\hline 7 & 20 & 1 & 1.5721 & 851591 & 1 & 3.8207 & 369846 & 0 & 0.0043 & 399 \\
\hline 8 & 20 & 1 & 6.3352 & 3539729 & 1 & 23.7996 & 2493203 & 0 & 0.0023 & 207 \\
\hline 9 & 20 & 1 & 18.3314 & 11294580 & 4 & 33.2138 & 2868979 & 0 & 0.0017 & 136 \\
\hline 10 & 20 & 3 & 22.5185 & 13536735 & 5 & 52.0812 & 4848676 & 0 & 0.0010 & 84 \\
\hline \multicolumn{11}{|c|}{ Weakly Correlated Instances } \\
\hline 2 & 20 & 0 & 0.0040 & 781 & 0 & 0.0000 & 26 & 0 & 0.0340 & 87 \\
\hline 3 & 20 & 0 & 0.0127 & 2693 & 0 & 0.0167 & 638 & 0 & 0.0117 & 240 \\
\hline 4 & 20 & 0 & 0.0327 & 6626 & 0 & 0.0390 & 1708 & 0 & 0.0133 & 379 \\
\hline 5 & 20 & 0 & 0.2363 & 53324 & 0 & 0.2917 & 12701 & 0 & 0.0107 & 521 \\
\hline 6 & 20 & 0 & 0.2763 & 65216 & 0 & 0.4220 & 20165 & 0 & 0.0080 & 405 \\
\hline 7 & 20 & 0 & 0.6237 & 168974 & 0 & 1.3180 & 60890 & 0 & 0.0050 & 245 \\
\hline 8 & 20 & 0 & 11.0927 & 2922499 & 2 & 6.5793 & 40339 & 0 & 0.0027 & 144 \\
\hline 9 & 20 & 0 & 27.5807 & 11263928 & 3 & 22.5878 & 1274756 & 0 & 0.0013 & 66 \\
\hline 10 & 20 & 2 & 25.1029 & 10697165 & 5 & 72.3472 & 4478185 & 0 & 0.0013 & 43 \\
\hline \multicolumn{11}{|c|}{ Strongly Correlated Instances } \\
\hline 2 & 20 & 0 & 0.0723 & 5747 & 0 & 0.0037 & 32 & 0 & 0.0743 & 118 \\
\hline 3 & 20 & 0 & 0.1243 & 6748 & 0 & 0.0053 & 65 & 0 & 0.0417 & 278 \\
\hline 4 & 20 & 0 & 0.1237 & 8125 & 0 & 0.0120 & 156 & 0 & 0.0353 & 331 \\
\hline 5 & 20 & 0 & 0.1777 & 14823 & 0 & 0.0360 & 563 & 0 & 0.0350 & 359 \\
\hline 6 & 20 & 0 & 0.1807 & 18012 & 0 & 0.0550 & 734 & 0 & 0.0217 & 194 \\
\hline 7 & 20 & 0 & 5.1393 & 882080 & 1 & 0.0928 & 1339 & 0 & 0.0137 & 109 \\
\hline 8 & 20 & 2 & 11.2361 & 2616615 & 3 & 12.2581 & 225236 & 0 & 0.0073 & 42 \\
\hline 9 & 20 & 2 & 20.0136 & 3150848 & 4 & 33.5415 & 693398 & 0 & 0.0060 & 25 \\
\hline 10 & 20 & 4 & 38.8554 & 8141902 & 9 & 34.3291 & 634358 & 0 & 0.0057 & 21 \\
\hline \multicolumn{11}{|c|}{ Subset-Sum Instances } \\
\hline 2 & 20 & $\overline{0}$ & 0.0820 & 2061 & 0 & 0.0033 & 9 & 0 & 0.0447 & 64 \\
\hline 3 & 20 & 0 & 0.2057 & 7033 & 0 & 0.0337 & 77 & 0 & 0.0310 & 248 \\
\hline 4 & 20 & 0 & 0.1760 & 8371 & 0 & 0.0503 & 137 & 0 & 0.0260 & 294 \\
\hline 5 & 20 & 0 & 0.2507 & 17514 & 0 & 0.1780 & 830 & 0 & 0.0257 & 315 \\
\hline 6 & 20 & 0 & 0.2393 & 17546 & 0 & 0.1953 & 738 & 0 & 0.0173 & 176 \\
\hline 7 & 20 & 0 & 5.7657 & 852615 & 1 & 0.3221 & 1752 & 0 & 0.0113 & 102 \\
\hline 8 & 20 & 2 & 14.9261 & 2546834 & 3 & 19.5930 & 270841 & 0 & 0.0073 & 39 \\
\hline 9 & 20 & 2 & 25.9393 & 3124421 & 4 & 41.8962 & 677467 & 0 & 0.0063 & 25 \\
\hline 10 & 20 & 4 & 51.5027 & 7907407 & 9 & 39.7500 & 627688 & 0 & 0.0057 & 20 \\
\hline
\end{tabular}

Table 2: MTM, Mulknap, and Bin Completion with Nogood Dominance Pruning on small MKP instances with varying $n / m$ ratio. The fail column indicates the number of instances (out of 30) that were not solved within the time limit (300 seconds/instance). The time (seconds on $2.7 \mathrm{GHz}$ Pentium 4) and nodes columns show average time spent and nodes generated on the successful runs, excluding the failed runs. 
average time spent and nodes generated on the successful runs, excluding the failed runs. All experiments described in this section were run on a $2.7 \mathrm{GHz}$ Pentium 4.

Our data confirms the observations of Pisinger (1999) and Martello and Toth (1981) that the class of uniform, random instances that require the most search for previous branchand-bound solvers appear to be generated when $n / m$ is relatively low. In other words, the $n / m$ ratio for the MKP appears to be a critical parameter that determines search difficulty, similar to the clause-to-variable ratio for satisfiability problems (Mitchell, Selman, \& Levesque, 1992). Table 2 shows that Mulknap and MTM have great difficulty with relatively small problems with small $n / m$ ratios, while bin completion could solve these problems very quickly.

Next, we investigated larger problem instances, where $n / m$ varied between 2 and 4 . In this experiment, we also included bin completion with nogood pruning $(\mathrm{BC}+\mathrm{NP})$, in addition to MTM, Mulknap, and BC+NDP.

As shown in Table 3 , for problems where $n / m=2$ or 3 , both variants of bin completion $(\mathrm{BC}+\mathrm{NP}$ and $\mathrm{BC}+\mathrm{NDP})$ dramatically outperformed both MTM and Mulknap, with the difference in performance becoming more pronounced as problem size was increased. Because the runs were truncated at 300 seconds per instance per algorithm, it is not possible to compare the full runtimes for all of the sets. Note, for example, that on subset-sum instances where $n=40$ and $m=20$, the mean runtime for $\mathrm{BC}+\mathrm{NDP}$ is 0.06 seconds, while neither MTM nor Mulknap solved any of the instances within 300 seconds each, so they are both at least three orders of magnitude slower than BC+NDP on these instances. We tried running the solvers longer in order to get actual runtimes (as opposed to a truncated, lower bound on runtimes), but found that even allocating an hour per problem was insufficient to allow MTM and Mulknap to solve any of these problems. These results suggest that bin completion is asymptotically more efficient than MTM and Mulknap for this class of problem instances. For the problems with $n / m=4$, the results are similar, with the notable exception that for the strongly-correlated instances, Mulknap outperformed bin completion when $n=40$ and $m=10$.

For problems where $n / m=2$ and 3 , we observed that BC+NDP consistently outperforms $\mathrm{BC}+\mathrm{NP}$ by a significant margin with respect to the number of nodes searched, and significant improvements in success rate and execution times are observed for the larger problems sets. However, when $n / m=4, \mathrm{BC}+\mathrm{NDP}$ still searches fewer nodes than $\mathrm{BC}+\mathrm{NP}$, but the difference is much less significant, and in fact, the reduced search is not enough to offset the increased overhead per node for NDP, so that the runtimes of $\mathrm{BC}+\mathrm{NP}$ and $\mathrm{BC}+\mathrm{NDP}$ end up being comparable (Table 3).

We also ran some experiments with instances with larger $n / m$ ratios. When $n / m \geq 5$, Mulknap clearly dominates all other current algorithms, solving instances with little or no search in under a second, as first demonstrated by Pisinger (1999). At high $n / m$ ratios, the bound-and-bound approach of Mulknap has a very high probability of finding an upper bound that matches the lower bound at each node, essentially eliminating the need for search. In fact, Pisinger (1999) showed that for instances with $n / m \geq 10$, there were almost no instances that required searching more than one node. 


\begin{tabular}{|c|c|c|c|c|c|c|c|c|c|c|c|c|c|}
\hline & & \multicolumn{3}{|c|}{ MTM } & \multicolumn{3}{|c|}{ Mulknap } & \multicolumn{3}{|c|}{ Bin Completion+NP } & \multicolumn{3}{|c|}{ Bin Completion + NDP } \\
\hline $\begin{array}{r}\mathrm{m} \\
\text { \# bins }\end{array}$ & $\begin{array}{r}\mathrm{n} \\
\text { \# items }\end{array}$ & fail & time & nodes & fail & time & nodes & fail & time & nodes & fail & time & nodes \\
\hline \multicolumn{14}{|c|}{ Uncorrelated Instances } \\
\hline 15 & 30 & 30 & - & - & 30 & - & - & 0 & 0.04 & 1215 & 0 & 0.02 & 887 \\
\hline 20 & 40 & 30 & - & - & 30 & - & - & 0 & 1.48 & 66681 & 0 & 0.72 & 26459 \\
\hline 25 & 50 & 30 & - & - & 30 & - & - & 4 & 72.66 & 2767939 & 3 & 32.43 & 1060723 \\
\hline 10 & 30 & 12 & 47.77 & 9240426 & 11 & 29.96 & 1649062 & 0 & 0.71 & 48407 & 0 & 0.61 & 33141 \\
\hline 15 & 45 & 30 & & - & 30 & - & - & 15 & 66.81 & 3369997 & 15 & 60.54 & 2516681 \\
\hline 5 & 20 & 0 & 0.08 & 32602 & 0 & 0.10 & 8487 & 0 & 0.01 & 253 & 0 & 0.01 & 237 \\
\hline 10 & 40 & 7 & 99.13 & 15083247 & 4 & 51.66 & 1744911 & 0 & 11.58 & 360567 & 0 & 13.47 & 297225 \\
\hline \multicolumn{14}{|c|}{ Weakly Correlated Instances } \\
\hline 15 & 30 & 30 & - & - & 30 & - & - & 0 & 0.07 & 2123 & 0 & 0.05 & 1050 \\
\hline 20 & 40 & 30 & - & - & 30 & - & - & 0 & 0.79 & 22553 & 0 & 0.35 & 7473 \\
\hline 25 & 50 & 30 & - & - & 30 & - & - & 0 & 34.39 & 691645 & 0 & 15.80 & 231934 \\
\hline 10 & 30 & 16 & 68.80 & 8252155 & 15 & 69.65 & 1647576 & 0 & 0.94 & 34001 & 0 & 0.79 & 25764 \\
\hline 15 & 45 & 30 & - & - & 30 & - & - & 19 & 52.42 & 1126242 & 18 & 67.17 & 1207099 \\
\hline 5 & 20 & 0 & 0.10 & 21150 & 0 & 0.14 & 5758 & 0 & 0.01 & 407 & 0 & 0.01 & 396 \\
\hline 10 & 40 & 29 & 205.99 & 12672965 & 27 & 131.41 & 2390152 & 0 & 21.10 & 565036 & 0 & 20.63 & 490983 \\
\hline \multicolumn{14}{|c|}{ Strongly Correlated Instances } \\
\hline 15 & 30 & 30 & - & - & 30 & - & - & 0 & 0.04 & 415 & 0 & 0.03 & 194 \\
\hline 20 & 40 & 30 & - & - & 30 & - & - & 0 & 3.46 & 5407 & 0 & 2.29 & 2694 \\
\hline 25 & 50 & 30 & - & - & 30 & - & - & 9 & 52.26 & 58500 & 4 & 58.72 & 61900 \\
\hline 10 & 30 & 1 & 23.48 & 2916858 & 0 & 7.02 & 88737 & 0 & 0.53 & 5662 & 0 & 0.50 & 5381 \\
\hline 15 & 45 & 30 & & - & 30 & - & - & 24 & 105.90 & 964566 & 23 & 118.72 & 1150024 \\
\hline 5 & 20 & 0 & 0.06 & 12490 & 0 & 0.02 & 486 & 0 & 0.01 & 264 & 0 & 0.01 & 258 \\
\hline 10 & 40 & 13 & 115.38 & 5528847 & 0 & 44.55 & 361987 & 2 & 51.89 & 263462 & 2 & 50.51 & 254094 \\
\hline \multicolumn{14}{|c|}{ Subset-Sum Instances } \\
\hline 15 & 30 & 30 & - & - & 30 & - & - & 0 & 0.01 & 171 & 0 & 0.01 & 95 \\
\hline 20 & 40 & 30 & - & - & 30 & - & - & 0 & 0.13 & 1664 & 0 & 0.06 & 806 \\
\hline 25 & 50 & 30 & - & - & 30 & - & - & 0 & 4.37 & 55334 & 0 & 1.59 & 19491 \\
\hline 10 & 30 & 0 & 25.58 & 1993648 & 0 & 4.74 & 43734 & 0 & 0.12 & 1816 & 0 & 0.12 & 1752 \\
\hline 15 & 45 & 18 & 0.0 & 27.58 & 23 & 100.19 & 480014 & 2 & 45.07 & 597581 & 2 & 43.44 & 562129 \\
\hline 5 & 20 & 0 & 0.05 & 5676 & 0 & 0.01 & 207 & 0 & 0.01 & 140 & 0 & 0.01 & 137 \\
\hline 10 & 40 & 5 & 35.20 & 946934 & 0 & 10.48 & 58317 & 0 & 1.98 & 26674 & 0 & 2.00 & 26097 \\
\hline
\end{tabular}

Table 3: Multiple knapsack problem results: Comparison of MTM, Mulknap, Bin Completion with Nogood Pruning (NP), and Bin Completion with Nogood Dominance Pruning (NDP) on hard MKP instances. The fail column indicates the number of instances (out of 30) that were not solved within the time limit (300 seconds/instance). The time (seconds on $2.7 \mathrm{GHz}$ Pentium 4) and nodes show average time spent and nodes generated on the successful runs, excluding the failed runs. 
On the other hand, for $n / m \geq 5$, bin completion tends to generate a very large (more than 10000) number of undominated bin assignments at each decision node, and runs out of memory while allocating the set of undominated bin assignments that are the candidates to be assigned to the current bin. While hybrid incremental branching (Section 3.4) eliminates this problem and allows the algorithm to run to completion, it is still not competitive with Mulknap (runs do not complete within a 300-second time limit).

We conclude that uniform instances of the multiple knapsack problem exhibit a "bimodal" characteristic, for which bin completion and bound-and-bound are complementary approaches. For $n / m \leq 4$, uniform MKP instances require a significant amount of search to solve, and our bin completion approach is clearly the current state of the art, as demonstrated by Table 3. On the other hand, for $n / m \geq 5$, the bound-and-bound approach as exemplified by Pisinger's Mulknap algorithm is the state of the art, and the runtimes are dominated by the computation of a single lower bound and a single upper bound at the root node. There is a rather sharp "phase transition" around $n / m=4$ where the dominant approach changes between bin completion and bound-and-bound (see Fukunaga, 2005 for details).

Because of the highly complementary nature of Mulknap and bin completion, the MKP is a natural candidate for the application of an algorithm portfolio approach (Huberman, Lukose, \& Hogg, 1997; Gomes \& Selman, 2001) where both Mulknap and bin completion are run in parallel on the same problem instance. Even under a trivial portfolio resource allocation scheme where both processes received equal time, the resulting total CPU usage of the portfolio on each instance would be no worse than twice that of the faster algorithm. Another way to combine bin completion with bound-and-bound is to add bound-and-bound at each node of the bin completion search, and is an avenue for future work.

\section{Min-Cost Covering Problem (MCCP)}

Given a set of containers with quotas and a set of items (characterized by a weight and cost), the objective of the MCCP is to assign items to containers such that all the container quotas are satisfied, and the sum of the costs of the items assigned to the containers is minimized (see 1.1.4 for a formal definition).

\subsection{Christofides, Mingozzi, and Toth (CMT) Algorithm}

The previous state-of-the-art algorithm for the min-cost covering problem is an early version of bin completion by Christofides, Mingozzi, and Toth (1979). Their algorithm is a bin completion algorithm which uses the CMT dominance criterion (see Section 2.3).

An effective lower bound can be computed by solving $m$ independent minimization problems (similar to the standard 0-1 Knapsack, except that the objective is to satisfy a container's quota while minimizing the cost of the items assigned to it), one for each of the bins, and then summing the results. This $L_{2}$ lower bound is a relaxation of the constraint that each item can only be used once.

Christofides et al. (1979) proposed several more complex lower bounds, but they found empirically that among all of the proposed lower bounds, the $L_{2}$ bound resulted in the best performance by far across a wide range of problem instances. 


\subsection{Bin Completion for the MCCP}

Our new bin completion algorithm for the MCCP is similar to the Christofides et al. algorithm. The major difference is that we use a more powerful dominance criterion (Proposition 5). Each node in the depth-first, search tree represents a minimal, feasible bin assignment for a particular bin. Our bin completion algorithm assigns bins in order of non-decreasing size, i.e., a smallest-bin-first variable ordering heuristic. We evaluated eight different strategies for ordering the candidate undominated bin assignments, and found that a min-weight strategy which sorts assignments in non-decreasing order of weight performed best. Fukunaga (2005) provides a detailed comparison of variable and value orderings for the MCCP. We use the same $L_{2}$ bound as the CMT algorithm.

\subsection{Experimental Results}

We compared the performance of bin completion variants and previous algorithms. We implemented the following algorithms.

- CMT - The Christofides, Mingozzi and Toth algorithm described above. Our implementation used smallest-bin-first variable ordering, min-weight value ordering, and the $L_{2}$ lower bound.

- CMT+NP - The CMT algorithm extended with nogood pruning.

- BC - Bin completion using min-weight value ordering and smallest-bin-first variable ordering.

- BC+NP - Bin completion with nogood pruning

- BC+NDP - Bin completion with nogood dominance pruning,

We used smallest-bin-first variable ordering for the CMT and BC variants after observing that this led to good performance compared with random or largest-bin-first variable orderings.

In the experiment shown in Table 4, we compared CMT, CMT+NP, BC, BC+NP, and $\mathrm{BC}+\mathrm{NDP}$. The purpose of this experiment was to evaluate the relative impact of each component of bin completion, by comparing various combinations of: (1) the type of dominance criterion used, (2) whether nogood pruning was used, and (3) whether nogood dominance pruning was used.

We used the same four classes of test problems (uncorrelated, weakly correlated, strongly correlated, and subset-sum) as for the multiple knapsack problem experiments (Section 4.4), with item weights and costs in the range $[10,1000]$. For each of the four problem classes, 30 instances were generated for various values of $m$ and $n$. We ran each algorithm on each instance. All experiments were run on a $2.7 \mathrm{GHz}$ Pentium 4. Each algorithm was given a time limit of 300 seconds to solve each instance. The fail column indicates the number of instances (out of 30) which could not be solved by the algorithm within the time limit. The time and nodes column show the total time spent and nodes generated, excluding the failed runs. 


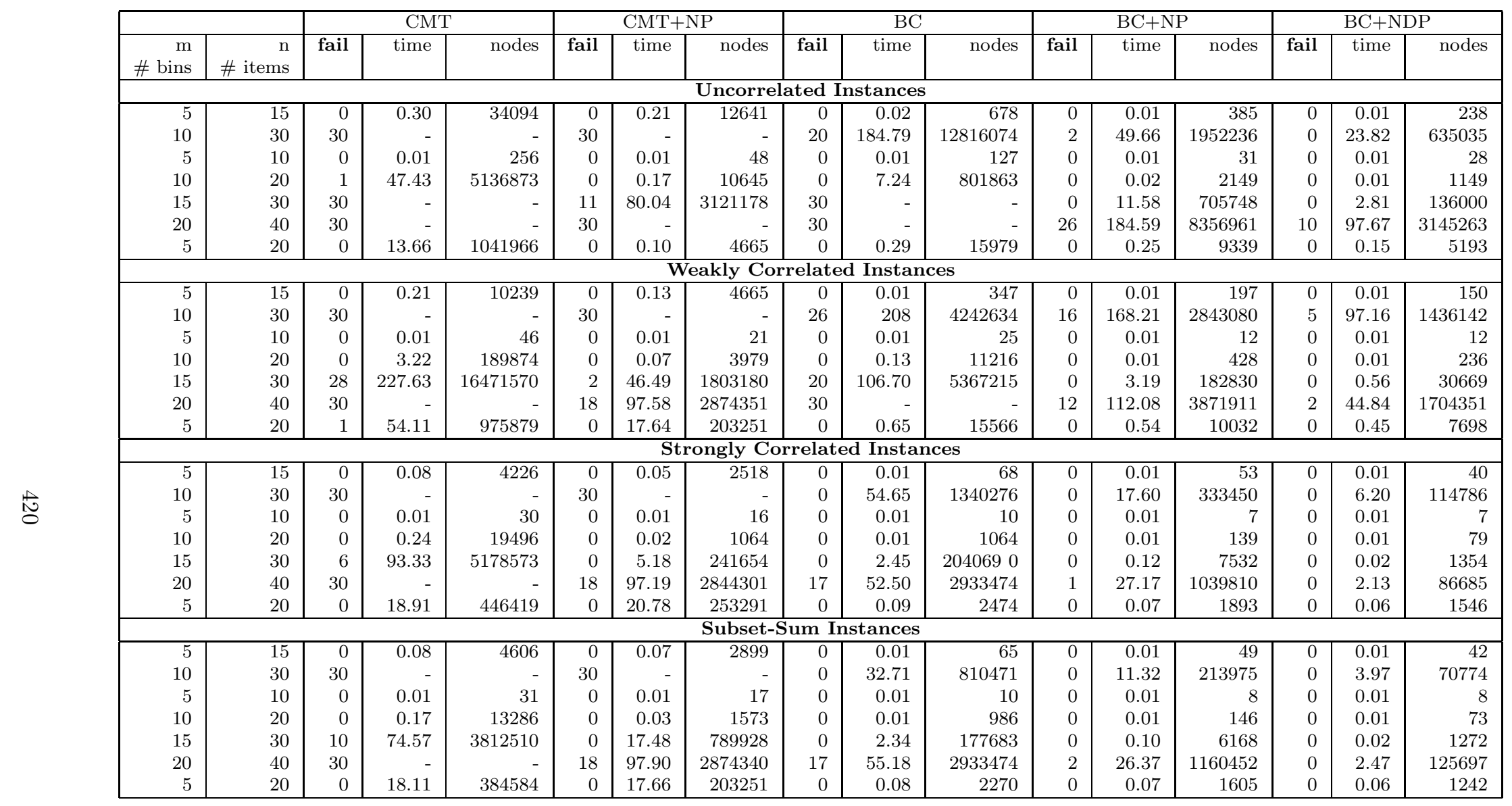

Table 4: Min-cost covering problem results: Comparison of (a) Christofides, Mingozzi, and Toth (CMT) algorithm, (b) CMT algorihm with our Nogood Pruning (NP), (c) Bin Completion, (d) Bin Completion with Nogood Pruning (NP), and (e) Bin Completion with Nogood Dominance Pruning (NDP). The fail column indicates the number of instances (out of 30) that were not solved within the time limit. The time (seconds on $2.7 \mathrm{GHz}$ Pentium 4) and nodes columns show average time spent and nodes generated on the successful runs, excluding the failed runs. 
As shown in Table 4, each component of bin completion has a significant impact. Although our dominance criterion requires much more computation per node than the simpler CMT criterion, the search efficiency is dramatically improved. Thus, BC performed much better than $\mathrm{CMT}$, and $\mathrm{BC}+\mathrm{NP}$ performed much better than $\mathrm{CMT}+\mathrm{NP}$.

Furthermore, the nogood pruning (NP) strategy significantly improves performance for both the algorithm based on the CMT dominance criterion, as well as our dominance criterion, as evidenced by the improvement of $\mathrm{CMT}+\mathrm{NP}$ over $\mathrm{CMT}$ and the improvement of $\mathrm{BC}+\mathrm{NP}$ over BC. In fact, nogood pruning is sufficiently powerful that it allows $\mathrm{CMT}+\mathrm{NP}$ to sometimes outperform pure BC (without nogood pruning). Thus, this data illustrates the power of nogood pruning, confirming similar results for bin packing reported in (Korf, 2003), as well as in some preliminary experiments for the MKP and MCCP.

Finally, BC+NDP results in the best performance, significantly outperforming BC+NP for the larger instances with respect to the number of problems solved within the time limit, as well as the runtimes and nodes on the problems that were solved.

We also implemented two baseline algorithms: a straightforward integer programming model using the freely available GNU glpk integer programming solver, as well as an itemoriented branch-and-bound algorithm which uses the $L_{2}$ lower bound, but these baselines performed very poorly compared to the CMT and bin completion algorithms (Fukunaga, 2005).

\section{The Bin Covering Problem}

Given a set of identical containers with quota $q$ and a set of $n$ items, each with weight $w_{i}$, the bin covering problem, sometimes called dual bin packing, is to assign all items to containers such that the number of containers whose quotas are satisfied (i.e., the sum of item weights assigned to the container equal or exceed the quota) is maximized (see 1.1.3 for formal definition). Although there has been considerable interest in the bin covering problem in the algorithm and operations research communities, most of the previous work on bin covering has been theoretical, focusing on approximation algorithms or heuristic algorithms (e.g., Assmann et al., 1984; Foster \& Vohra, 1989; Csirik, Frenk, Galambos, \& Kan, 1991; Csirik, Johnson, \& Kenyon, 2001), and analysis of the properties of some classes of instances (e.g., Rhee \& Talagrand, 1989).

\subsection{The Labbé, Laporte, Martello Algorithm}

The state-of-the-art algorithm for opimally solving bin covering is an item-oriented branchand-bound algorithm by Labbé, Laporte, and Martello (1995). We refer to this as the $L L M$ algorithm. The items are sorted in non-increasing order of size. Each node represents a decision as to which bin to put an item into. At each node, upper bounds based on combinatorial arguments are computed, and the remaining subproblem is reduced using two reduction criteria. At the root node, a set of heuristics is applied in order to compute an initial solution and lower bound. The LLM upper and lower bounds are described in (Labbé et al., 1995). 


\subsection{Bin Completion for Bin Covering}

Our bin completion algorithm for bin covering works as follows. First, we use the LLM upper bounding heuristics at the root node to find an initial solution and lower bound. Then, we apply a bin completion branch-and-bound algorithm, using our new dominance criterion (Proposition 3). Each node in the depth-first, search tree represents a minimal, feasible bin assignment for a particular bin that include the largest remaining item. At each node, we apply the same upper bounding procedures as the LLM algorithm to compute an upper bound. In addition, we apply the LLM reduction criteria at each node. We evaluated eight different strategies for ordering undominated bin assignments, and found that the mincardinality-min-sum strategy (sort bin assignments in order of non-decreasing cardinality, breaking ties by non-decreasing sum) performed best overall (Fukunaga, 2005).

\subsection{Empirical Results}

In order to evaluate our bin covering algorithm, we considered the class of uniform, random problem instances previously studied by Labbé, Laporte, and Martello. This is a simple model in which $n$ items are chosen uniformly in the range [min, max], where max is less than the bin quota $q$. In their experimental evaluations, Labbé, Laporte, and Martello used items with weights in the range 1 and 100, and bin quotas ranging between 100 and 500 .

However, many instances in this class can be solved without any search. If the LLM lower bound heuristics find a solution with the same number of bins as the LLM upper bound, then we have found the optimal solution and can terminate without any search. We say that an instance is trivial if it is solved without any search, and nontrivial otherwise.

To illustrate the prepondrance of trivial instances, we generated 10000 uniform, random instances with bin quota 100000 and 120 items with weights in the range [1,99999]. Of these, 9084 were solved at the root node. This shows that most uniform random bin covering instances are, in fact, trivial given powerful upper and lower bounds. We have previously observed a similar phenomenon for bin packing - most uniform, random bin packing instances are solved at the root node by the combination of the best-first-decreasing heuristic and the Martello-Toth L2 lower bound (Korf, 2002).

It is well-known that the number of significant digits of precision in the weights of the items significantly affects the difficulty of one-dimensional packing problems such as the 0-1 Knapsack problem (Kellerer et al., 2004). In general, problem difficulty increases with precision. This property extends to multicontainer, one-dimensional packing problems as well (e.g., Pisinger, 1999). We confirmed that problem difficulty was highly correlated with the number of significant digits of item weights (Fukunaga, 2005).

Therefore, in the experiments described below, we only used nontrivial instances, in order to highlight the impact of differences in search strategy. That is, when generating test instances, we filtered out all trivial instances by testing whether the LLM upper bound is matched by its lower bound heuristics. Furthermore, we use high precision problems (quotas of 10000 or more) in order to focus on the most difficult instances.

\subsubsection{Hybrid InCREmental Branching}

In Section 3.4, we proposed hybrid incremental branching, a strategy for avoiding the runtime and memory overheads imposed by completely enumerating all undominated children 
of a node. In our earlier experiments with the multiple knapsack and min-cost covering problems, (Sections 4-5), the problem instances used in our experiments had fewer than 50 items, and we have not observed more than a few hundred assignments generated at each node in our MKP and MCCP experiments. Thus, the number of candidate undominated bin assignments generated per node did not become a bottleneck, and hybrid incremental branching was unnecessary. However, as (1) the average number of items that fit in a container increases, and (2) the number of items increases, the number of candidate undominated bin assignments per node increases. Therefore, the benchmark comparisons below for bin covering, hybrid incremental branching becomes much more relevant.

To illustrate this, we performed the following experiment. We generated 20 nontrivial instances with bin quota $q=20000$ and 100 items in the range [1,9999]. We applied bin completion + NDP to these problem instances, using hybrid incremental branching with various values for the parameter $h$, which limits the number of children that are generated at once at every node. For $h=2000,200,20$, and 2, the instances were solved on average in 4.998 seconds, 0.150 seconds, 0.0139 seconds, and 0.0079 seconds, respectively. The average number of nodes expanded was 20.2 for all $h \in\{2,20,200,2000\}$. In other words, these instances were easily solved by relying on the leftmost children at each node in the bin completion search tree - generating additional children was an entirely unnecessary but very expensive overhead. Thus, as $h$ increases, the same number of nodes are being explored, but each node requires much more computation to enumerate and sort up to $h$ undominated children, resulting in more than two orders of magnitude difference in runtime between $h=2$ and $h=2000$. To see how large $h$ would have to be in order to be able to enumerate all of the undominated children of a node, we have experimented with $h$ up to 10000 , but found it insufficient (the statically allocated array of size $h$ overflowed during the runs).

We experimented with several values of $h$ on several classes of problems, but found that the optimal value of $h$ varied significantly depending on the problem instance. In our experiments below, we use hybrid incremental branching with $h=100$, in order to set a balance between minimizing the number of nodes expanded (exploiting value ordering among the children of each node) and minimizing the computational overhead per node (by minimizing the number of children generated).

\subsubsection{Comparing LLM and Bin Completion}

Next, we compared LLM, bin completion+NP, and bin completion+NDP using larger instances. We also implemented a straightforward integer linear programming model using GNU glpk for bin covering, but found that it performed much worse than the LLM and bin completion algorithms (Fukunaga, 2005).

For each $n \in\{60,80,100\}$, we generated 2000 non-trivial, uniform random instances where the items were chosen uniformly in the range [1,99999], and the bin quota was 100000 . We also generated 2000 non-trivial instances where $n=100, q=200000$, and items were in the range $[1,99999]$. We ran our implementations of the three algorithms on each instance, with a time limit of 180 seconds per instance. As shown in Table 5, the bin completion algorithms significantly outperformed LLM. On the harder problems, bin completion + NDP significantly outperforms bin completion + NP. For the problems with $n=100, q=200000$, 


\begin{tabular}{|r|r|r|r|r|r|r|r|r|r|r|r|}
\hline & & \multicolumn{3}{|c|}{ Labbé et al. } & \multicolumn{2}{|c|}{ Bin Completion+NP } & \multicolumn{2}{|c|}{ Bin Completion+NDP } \\
\hline $\mathrm{n}$ (\# of items) & q (quota) & fail & time & nodes & fail & time & nodes & fail & time & nodes \\
\hline \multicolumn{8}{|c|}{ 2000 nontrivial instances per set } \\
\hline 60 & 100000 & 10 & 0.39 & 25196 & 6 & 0.08 & 33287 & 2 & 0.11 & 13735 \\
80 & 100000 & 36 & 1.02 & 62093 & 15 & 0.18 & 39740 & 12 & 0.09 & 10517 \\
100 & 100000 & 45 & 1.98 & 113110 & 15 & 0.42 & 152113 & 8 & 0.31 & 31107 \\
\hline \multicolumn{8}{|c|}{100 nontrivial instances per set } \\
\hline 100 & 200000 & 100 & - & - & 3 & 0.11 & 25 & 3 & 0.11 & 25 \\
\hline
\end{tabular}

Table 5: Bin Covering results: Comparison of Labbé, Laporte, and Martello algorithm, Bin Completion with Nogood Pruning, and Bin Completion with Nogood Dominance Pruning. The fail column indicates the number of instances (out of 30) that were not solved within the time limit. The time (seconds on $2.7 \mathrm{GHz}$ Pentium 4) and nodes columns show average time spent and nodes generated on the successful runs, excluding the failed runs.

our results indicate that while these instances are extremely difficult for the LLM algorithm, which failed to solve any of the 100 instances within the time limit, these problems are actually relatively easy for bin completion. Since little or no search is being performed by bin completion, bin completion+NDP does not improve upon bin completion+NP.

\section{Bin Packing}

The bin completion approach was originally developed for the bin packing problem, and was shown to significantly outperform the Martello-Toth Procedure on randomly generated instances (Korf, 2002, 2003). We implemented an extended bin completion algorithm for the bin packing problem, and summarize our results below. For further details, see (Fukunaga, 2005). Our bin completion based solver incorporated nogood dominance pruning and a min-cardinality-max-weight value ordering strategy, in which (1) completions are sorted in order of non-decreasing cardinality, and (2) ties are broken according to non-increasing weight.

We evaluated our bin completion solver using the set of standard OR-LIB instances. ${ }^{6}$ This test set consists of 80 "triplet" instances (where the elements are generated three at a time so that the sum of the elements add exactly to the bin capacity) and 80 "uniform" instances (where items sizes are chosen from a uniform random distribution). The bin completion solver (executed on a $1.3 \mathrm{GHz}$ Athlon) was given a time limit of 15 minutes on each instance from the benchmark set. It solved all 80 of the triplet instances from ORLIB within 1500 seconds combined. It solved all 40 of the uniform instances with 120 and 250 items in under 4 seconds combined. It solved 18 (out of 20) of the 500-item uniform instances in 11.13 seconds combined, but failed to solve 2 instances. Bin completion solved 19 (out of 20) of the 1000-item uniform instances in 44 seconds combined, but failed to solve one of the instances.

6. available at http://www.brunel.ac.uk/depts/ma/research/jeb/info.html 
However, our bin completion solver was not competitive with the state of the art, which is a recently developed branch-and-price integer linear programming solver by Belov and Scheithauer (2006). Belov and Scheithauer provide a new benchmark set of 28 very hard bin packing instances; their solver solved most of these within seconds, although some took hours. Our current bin completion code could not solve any of the 28 instances, given 15 minutes per instance. They also report that the largest triplet instances from OR-LIB (Triplet-501) were solved in an average of 33 seconds per instance (660 seconds total) on a $1 \mathrm{GHz}$ AMD Athlon XP. Furthermore, Belov was kind enough to run their solver on a set of one hundred, 80-item, uniform, random instances that we generated with items in the range $[1,1000000]$ and a bin capacity of 1000000 . Their solver solved all of these instances in less than 1 second each at the root node (i.e., without any search), using rounding heuristics based on the linear programming LP solution, whereas our best bin completion solver required 534 seconds and searched 75,791,226 nodes.

\subsection{Branch-and-Price vs. Bin Completion}

Recent branch-and-price approaches for bin packing such as the solver by Belov and Scheithauer use a bin-oriented branching strategy, where decisions correspond to the instantiation of one or more maximal bin assignments (see Valério de Carvalho, 2002, for a survey of branch-and-price approaches). At each node, a column generation procedure is used to compute the LP lower bound. They derive much of their power from a very accurate LP lower bound based on a cutting-stock formulation of bin packing (Gilmore \& Gomory, 1961), which has been observed to almost always give the optimal value as the lower bound, and has never been observed to give a value that is more than one greater than the optimal value (e.g., Wäscher \& Gau, 1996). In addition, rounding heuristics applied to fractional LP-solutions often yield the optimal, integral solution. The combination of a very tight LP lower bound and good upper bounding procedure results in very little search being performed for almost all problem instances.

This branch-and-price LP-based approach does not seem to generalize straightforwardly to the MKP and MCCP, in part due to the differences in the granularity of the objective function. The objective function for bin packing counts the number of bins used. On the other hand, the objective functions for the MKP and MCCP sums the profits of the items assigned to the containers. Thus, the number of possible, distinct objective function values for the MKP and MCCP is much larger than the number of distinct objective function values for a bin packing problem of comparable size. Therefore, even if we assume the existence of some formulation analogous to the cutting-stock problem, it is not likely that rounding up the LP solution for the MKP, MCCP, or other problems with objective functions that are fine-grained compared to bin packing will result in an optimistic bound that is as accurate as for bin packing. This suggests that it may be difficult to develop a branch-and-price solver that is competitive for these problems. On the other hand, since the granularity of the objective function for bin covering is the same as that for bin packing, it is possible that

a branch-and-price approach could be applied to bin covering. However, we are unaware of any such approach in the literature. 


\section{Conclusions}

We studied bin completion, a branch-and-bound approach for multi-container packing, knapsack, and covering problems. While previous work focused on item-oriented, branchand-bound strategies that assign one item at a time to containers, bin completion is a bin-oriented branch-and-bound algorithm that uses a dominance relationship between bin assignments to prune the search. We presented a general framework for this approach, and showed its general utility and applicability to multicontainer problems. We proposed several extensions to bin completion that improve its efficiency, including nogood pruning, nogood dominance pruning, variable and value ordering heuristics, and hybrid incremental undominated completion generation. We demonstrated the power of the bin completion approach by developing new, state-of-the-art algorithms for three fundamental multicontainer problems. We showed that bin completion algorithms significantly outperform the Mulknap (Pisinger, 1999) and MTM (Martello \& Toth, 1981) and algorithms on hard MKP instances. We developed a new, state-of-the-art algorithm for the MCCP based on bin completion. We showed that by exploiting a more powerful dominance criterion, our new bin completion algorithms significantly outperform an early bin completion algorithm by Christofides, Mingozzi, and Toth (1979). We developed a new, state-of-the-art algorithm for bin covering based on bin completion, and showed that our bin completion algorithm significantly outperforms the item-oriented branch and bound algorithm by Labbé, Laporte, and Martello (1995). However, our results for bin packing were not competitive with the stateof-the-art solver based on the cutting-stock approach. We showed that for all four of the problems studied, nogood dominance pruning consistently improves upon the performance of bin completion with nogood pruning. ${ }^{7}$

While we have focused on four particular multicontainer problems in this paper, there are many similar problems involving the assignment of objects to multiple containers where similar dominance relationships between candidate bin assignments can be exploited. Examples include the generalized assignment problem, a widely studied generalization of the MKP with many applications where the weight and profit of an item is a function of the container to which it is assigned (e.g., Cattrysse \& Wassenhove, 1992; Martello \& Toth, 1990), multiprocessor scheduling, which is equivalent to $k$-way number partitioning, (Dell'Amico \& Martello, 1995), and the segregated storage problem (Neebe, 1987; Evans \& Tsubakitani, 1993). In addition, there are variants of the problems we studied with additional constraints, such as the class-constrained multiple knapsack problem (Shachnai \& Tamir, 2001a, 2001b; Kellerer et al., 2004) which has applications in multimedia file storage. Exploiting powerful dominance criteria in a bin completion framework appears to be a promising future direction for such multicontainer problems.

One issue with bin completion is that as the number of unique items grows, the number of undominated bin assignments grows rapidly. While we showed that hybrid incremental branching can significantly alleviate this problem (Section 6.3.1), a drawback is that it limits the utility of value-ordering heuristics that are applied to sort undominated bin assignments after they are generated. Thus, an algorithm that generates undominated assignments in

7. We previously showed that nogood pruning significantly improves performance over bin-completion without pruning in (Korf, 2003). This was also confirmed for the MCCP (Table 4), and for the MKP and bin covering in preliminary experiments. 
an order that conforms to a desired heuristic value ordering, rather than relying on sorting the assignments after they are all generated, is an area for future work.

\section{Acknowledgments}

Thanks to Gleb Belov for running his solver on some of our bin packing test instances. The anonymous reviewers provided many helpful comments and suggestions that improved this paper. This research was supported by NSF under grant No. EIA-0113313, and by the Jet Propulsion Laboratory, California Institute of Technology, under a contract with the National Aeronautics and Space Administration.

\section{References}

Assmann, S., Johnson, D., Kleitman, D., \& Leung, J. (1984). On a dual version of the one-dimensional binpacking problem. Journal of Algorithms, 5, 502-525.

Belov, G., \& Scheithauer, G. (2006). A branch-and-cut-and-price algorithm for onedimensional stock cutting and two-dimensional two-stage cutting. European Journal of Operational Research, 171, 85-106.

Caprara, A., Kellerer, H., \& Pferchy, U. (2000a). A PTAS for the multiple-subset sum problem with different knapsack capacities. Information Processing Letters, 73, 111118.

Caprara, A., Kellerer, H., \& Pferschy, U. (2000b). The multiple subset sum problem. SIAM Journal of Optimization, 11, 308-319.

Caprara, A., Kellerer, H., \& Pferschy, U. (2003). A 3/4-approximation algorithm for multiple subset sum. Journal of Heuristics, 9, 99-111.

Carlyle, M., Knutson, K., \& Fowler, J. (2001). Bin covering algorithms in the second stage of the lot to order matching problem. Journal of the Operational Research Society, 52, 1232-1243.

Cattrysse, D., \& Wassenhove, L. V. (1992). A survey of algorithms for the generalized assignment problem. European Journal of Operational Research, 60, 260-272.

Christofides, N., Mingozzi, A., \& Toth, P. (1979). Loading problems. In Christofides, N., Mingozzi, A., Toth, P., \& Sandi, C. (Eds.), Combinatorial Optimization. John Wiley \& Sons.

Csirik, J., Frenk, J., Galambos, G., \& Kan, A. R. (1991). Probabilistic analysis of algorithms for dual bin packing problems. Journal of Algorithms, 12, 189-203.

Csirik, J., Johnson, D., \& Kenyon, C. (2001). Better approximation algorithms for bin covering. In Proc. of the 12th ACM/SIAM Symposium on Discrete Algorithms, pp. $557-566$.

Dell'Amico, M., \& Martello, S. (1995). Optimal scheduling of tasks on identical parallel processors. ORSA Journal on Computing, 7(2), 181-200.

Eilon, S., \& Christofides, N. (1971). The loading problem. Management Science, 17(5), 259-268. 
Evans, J., \& Tsubakitani, S. (1993). Solving the segregated storage problem with Benders' partitioning. Journal of the Operational Research Society, 44(2), 175-184.

Fahle, T., Schamberger, S., \& Sellmann, M. (2001). Symmetry breaking. In Proceedings of the International Conference on Constraint Programming, pp. 93-107.

Focacci, F., \& Milano, M. (2001). Global cut framework for removing symmetries. In Proceedings of the International Conference on Constraint Programming, pp. 77-92.

Focacci, F., \& Shaw, P. (2002). Pruning sub-optimal search branch brances using local search. In Proc. Fourth International Workshop on Integration of AI and OR Techniques in Constraing Programming for Combinatorial Optimisation Problems (CPAI-OR), pp. 181-189.

Foster, D., \& Vohra, R. (1989). Probabilistic analysis of a heuristic for the dual bin packing problem. Information Processing Letters, 31, 287-290.

Fukunaga, A. (2005). Bin-Completion Algorithms for One Dimensional, Multicontainer Packing Problems. Ph.D. thesis, UCLA.

Gent, I., \& Smith, B. (2000). Symmetry breaking during search in constraint programming. In Proc. European Conference on Artificial Intelligence, pp. 599-603.

Gilmore, P., \& Gomory, R. (1961). A linear programming approach to the cutting stock problem. Operations Research, 9, 849-859.

Gomes, C., \& Selman, B. (2001). Algorithm portfolios. Artificial Intelligence, 126, 43-62.

Huberman, B., Lukose, R., \& Hogg, T. (1997). An economic approach to hard computational problems. Science, 265, 51-54.

Kalagnanam, J., Davenport, A., \& Lee, H. (2001). Computational aspects of clearing continuous call double auctions with assignment constraints and indivisible demand. Electronic Commerce Research, 1, 221-238.

Kellerer, H., Pferschy, U., \& Pisinger, D. (2004). Knapsack Problems. Springer-Verlag.

Korf, R. (2002). A new algorithm for optimal bin packing. In Proceedings of AAAI, pp. $731-736$.

Korf, R. (2003). An improved algorithm for optimal bin packing. In Proceedings of the International Joint Conference on Artificial Intelligence, pp. 1252-1258.

Labbé, M., Laporte, G., \& Martello, S. (1995). An exact algorithm for the dual bin packing problem. Operations Research Letters, 17, 9-18.

Labbé, M., Laporte, G., \& Martello, S. (2003). Upper bounds and algorithms for the maximum cardinality bin packing problem. European Journal of Operational Research, 149, 490-498.

Martello, S., \& Toth, P. (1977). An upper bound for the zero-one knapsack problem and a branch and bound algorithm. European Journal of Operational Research, 1, 169-175.

Martello, S., \& Toth, P. (1981). A bound and bound algorithm for the zero-one multiple knapsack problem. Discrete Applied Mathematics, 3, 275-288.

Martello, S., \& Toth, P. (1990). Knapsack problems: algorithms and computer implementations. John Wiley \& Sons. 
Mitchell, D., Selman, B., \& Levesque, H. (1992). Hard and easy distributions of SAT problems. In Proceedings of AAAI, pp. 459-65.

Neebe, A. (1987). An improved, multiplier adjustment procedure for the segregated storage problem. Journal of the Operational Research Society, 38(9), 815-825.

Pisinger, D. (1999). An exact algorithm for large multiple knapsack problems. European Journal of Operational Research, 114, 528-541.

Rhee, W., \& Talagrand, M. (1989). Optimal bin covering with items of random size. SIAM Journal on Computing, 18, 487-498.

Scholl, A., Klein, R., \& Jürgens, C. (1997). BISON: A fast hybrid procedure for exactly solving the one-dimensional bin packing problem. Computers in Operations Research, $24(7), 627-645$.

Shachnai, H., \& Tamir, T. (2001a). On two class-constrained versions of the multiple knapsack problem. Algorithmica, 29, 442-467.

Shachnai, H., \& Tamir, T. (2001b). Polynomial time approximation schemes for classconstrained packing problems. Journal of Scheduling, 4, 313-338.

Shaw, P. (2004). A constraint for bin packing. In Proceedings of 10th International Conference on the Principles and Practice of Constraint Programming 2004 (CP-2004), Lecture Notes in Computer Science Vol. 3258, pp. 648-662. Springer.

Valério de Carvalho, J. (2002). LP models for bin packing and cutting stock problems. European Journal of Operational Research, 141, 253-273.

Wäscher, G., \& Gau, T. (1996). Heuristics for the integer one-dimensional cutting stock problem: A computational study. OR Spektrum, 18(3), 131-144. 